\title{
Asymmetric Total Syntheses of (+)-Mycoepoxydiene and Related Natural Product (-)-1893A: Application of One-Pot Ring-Opening/Cross/Ring-Closing Metathesis to Construct their 9-Oxabicyclo[4.2.1]nona-2,4-diene Skeleton
}

Ken-ichi Takao, Hiroyuki Yasui, Shun Yamamoto, Daisuke Sasaki, Soujiro Kawasaki, Gohshi Watanabe, and Kin-ichi Tadano*

\section{Supporting Information}

\section{Contents}

Experimental procedures for new compounds $\quad \mathrm{S} 1$

NMR data of natural 1893A (2) and synthetic (-)-2 (Table S1) S18

${ }^{1} \mathrm{H}$ and ${ }^{13} \mathrm{C}$ NMR spectra of new compounds $\quad$ S19

General methods. Melting points are uncorrected. Specific rotations were measured in a $10 \mathrm{~mm}$ or $100 \mathrm{~mm}$ cell. ${ }^{1} \mathrm{H}$ NMR spectra were recorded at $270 \mathrm{MHz}$ or at $300 \mathrm{MHz}$ with tetramethylsilane as an internal standard. ${ }^{13} \mathrm{C}$ NMR spectra were recorded at $68 \mathrm{MHz}$ or at $75 \mathrm{MHz}$. All spectra were recorded in $\mathrm{CDCl}_{3}$ as solvent, unless otherwise described. Thin-layer chromatography (TLC) was performed on Merck Kieselgel $60 \mathrm{~F}_{254}$ plates. The crude reaction mixtures and extractive materials were purified by chromatography on Daisogel IR-60 (Daiso) or Wakogel C-300 (Wako). Unless otherwise described, reactions were carried out at ambient temperature. Combined organic extracts were dried over anhydrous $\mathrm{Na}_{2} \mathrm{SO}_{4}$. Solvents were removed from the reaction mixture or combined organic extracts by concentration under reduced pressure using an evaporator with bath at $35-45^{\circ} \mathrm{C}$.

$(1 S, 2 S, 3 R, 4 R)$-2-Acetoxymethyl-3-hydroxymethyl-7-oxabicyclo[2.2.1] hept-5-ene $((+)-10)$. The following reaction was carried out according Bloch's procedure. ${ }^{1}$ To a stirred solution of $\mathbf{9}^{2}(3.91 \mathrm{~g}, 25.0$ $\mathrm{mmol})$ in vinyl acetate $(78 \mathrm{~mL})$ was added lipase PS (Amano) $(0.782 \mathrm{~g})$. The mixture was stirred for $15 \mathrm{~h}$, and the enzyme was removed by filtration and washed with EtOAc. The combined filtrate and washings were concentrated in vacuo. The residue was purified by column chromatography on silica gel (EtOAc:hexane, 1:2) to provide $4.10 \mathrm{~g}(83 \%)$ of (+)-10 as white crystals: $\mathrm{mp} 49-51{ }^{\circ} \mathrm{C}$ (lit. ${ }^{1} \mathrm{mp} 44{ }^{\circ} \mathrm{C}$ ); TLC $R_{f} 0.17$ (EtOAc:hexane, 1:1); [ర] ${ }_{\mathrm{D}}{ }^{24}+8.1$ (c 2.16, MeOH) [lit. ${ }^{1}[\square]_{\mathrm{D}}{ }^{20}+7.8$ (c 1.0, MeOH)]; IR (KBr) 3400, 2940, $1740 \mathrm{~cm}^{-1}$; ${ }^{1} \mathrm{H}$ NMR (270 MHz) $\square 1.78$ (br, 1H, OH), 1.87-2.08 (m, 2H), 2.09 (s, 3H), $3.66(\mathrm{~m}, 1 \mathrm{H}), 3.86(\mathrm{~m}, 1 \mathrm{H}), 4.04(\mathrm{dd}, 1 \mathrm{H}, J=9.3,10.9 \mathrm{~Hz}), 4.31(\mathrm{dd}, 1 \mathrm{H}, J=5.5,10.9 \mathrm{~Hz}), 4.81(\mathrm{~s}, 1 \mathrm{H})$,

\footnotetext{
${ }^{1}$ Cinquin, C.; Schaper, I.; Mandville, G.; Bloch, R. Synlett 1995, 339-340.

2 Das, J.; Vu, T.; Harris, D. N.; Ogletree, M. L. J. Med. Chem. 1988, 31, 930-935.
} 
4.90 (s, 1H), 6.37-6.42 (m, 2H); ${ }^{13} \mathrm{C}$ NMR (68 MHz) $\square 21.0,39.2,42.0,61.7,64.2,80.4,80.5,135.3$, 135.7, 171.0; HRMS calcd for $\mathrm{C}_{10} \mathrm{H}_{14} \mathrm{O}_{4}\left(\mathrm{M}^{+}\right) \mathrm{m} / \mathrm{z}$ 198.0892, found 198.0899; HPLC analysis (column, Daicel Chiralcel OD+OD-H, $i$-PrOH:hexane $=1: 30$, flow rate $=0.5 \mathrm{~mL} / \mathrm{min}) ; t_{\mathrm{R}}(\mathrm{min})=30.1$ for the 4- $t$ butylbenzoate of $(-)-\mathbf{1 0}, 36.2$ for the 4-t-butylbenzoate of $(+)-\mathbf{1 0} .^{3}$ Compound $(+)-\mathbf{1 0}$ was determined to be $96 \%$ ee.

\section{$(1 R, 2 R, 3 S, 4 S)$-2-(t-Butyldiphenylsilyloxy)methyl-3-hydroxymethyl-7-oxabicyclo[2.2.1]hept-5-} ene $((+)-7)$. To a cooled $\left(0^{\circ} \mathrm{C}\right)$ stirred solution of $(+)-\mathbf{1 0}(4.79 \mathrm{~g}, 24.2 \mathrm{mmol})$ in DMF $(100 \mathrm{~mL})$ were added imidazole $(3.95 \mathrm{~g}, 58.6 \mathrm{mmol})$ and TBDPSCl $(10.1 \mathrm{~mL}, 39.1 \mathrm{mmol})$. The mixture was stirred for $23 \mathrm{~h}$, diluted with EtOAc $(200 \mathrm{~mL})$, and washed with $\mathrm{H}_{2} \mathrm{O}(200 \mathrm{~mL} \square 3)$. The organic layer was dried and concentrated in vacuo to provide crude silyl ether $(11.8 \mathrm{~g})$, which was used directly in the next step. In a small scale experiment, crude silyl ether was purified by column chromatography on silica gel (EtOAc:hexane, 1:6) and obtained as a colorless oil: TLC $R_{f} 0.87$ (EtOAc:hexane, 1:1); [ $]_{\mathrm{D}}{ }^{22}+6.8(c 1.87$, $\mathrm{CHCl}_{3}$ ); IR 2940, $1740 \mathrm{~cm}^{-1} ;{ }^{1} \mathrm{H}$ NMR (300 MHz) $\square 1.06$ (s, 9H), 1.84-1.94 (m, 2H), 1.98 (s, 3H), 3.65 $(\mathrm{dd}, 1 \mathrm{H}, J=9.4,9.5 \mathrm{~Hz}), 3.78(\mathrm{dd}, 1 \mathrm{H}, J=5.3,9.5 \mathrm{~Hz}), 3.89(\mathrm{dd}, 1 \mathrm{H}, J=10.2,10.5 \mathrm{~Hz}), 4.23(\mathrm{dd}, 1 \mathrm{H}, J=$ 4.7, 10.5 Hz), 4.77 (br s, 1H), 4.95 (br s, 1H), 6.34 (dd, 1H, J= 1.3, 5.8 Hz), 6.39 (dd, 1H, J = 1.3, 5.8 Hz), 7.36-7.46 (m, 6H), 7.65-7.69 (m, 4H); ${ }^{13} \mathrm{C}$ NMR (68 MHz) $\square$ 19.2, 20.9, $26.8 \square$ 3, 39.0, 42.5, 62.9, 64.0, 80.3, 80.4, $127.7 \square 4,129.7 \square 2,133.4,133.5,135.1,135.5 \square$ 4, 135.9, 170.8; HRMS calcd for $\mathrm{C}_{22} \mathrm{H}_{23} \mathrm{O}_{4} \mathrm{Si}\left(\mathrm{M}^{+}-t-\mathrm{C}_{4} \mathrm{H}_{9}\right) m / z$ 379.1366, found 379.1362 .

To a stirred solution of crude silyl ether obtained above $(11.8 \mathrm{~g})$ in $\mathrm{MeOH}(100 \mathrm{~mL})$ was added $\mathrm{NaOMe}$ (1.0 M solution in $\mathrm{MeOH}, 4.84 \mathrm{~mL}, 4.84 \mathrm{mmol}$ ). The mixture was stirred for $2 \mathrm{~h}$, diluted with saturated brine $(200 \mathrm{~mL})$, and extracted with EtOAc $(400 \mathrm{~mL} \square 3)$. The combined extracts were dried and concentrated in vacuo. The residue was purified by column chromatography on silica gel (EtOAc:hexane, 1:3) to provide $8.80 \mathrm{~g}(92 \%)$ of (+)-7 as white crystals: mp $65-67{ }^{\circ} \mathrm{C}$; TLC $R_{f} 0.38$ (EtOAc:hexane, 1:1); $[\square]_{\mathrm{D}}{ }^{21}+17.7\left(c 4.23, \mathrm{CHCl}_{3}\right)$; IR 3440, $2940 \mathrm{~cm}^{-1} ;{ }^{1} \mathrm{H}$ NMR (270 MHz) $\square 1.05(\mathrm{~s}, 9 \mathrm{H}), 1.91-2.00(\mathrm{~m}, 2 \mathrm{H})$, $2.90(\mathrm{br}, 1 \mathrm{H}, \mathrm{OH}), 3.67(\mathrm{dd}, 1 \mathrm{H}, J=5.9,11.0 \mathrm{~Hz}), 3.76(\mathrm{dd}, 1 \mathrm{H}, J=6.4,10.4 \mathrm{~Hz}), 3.84-3.91(\mathrm{~m}, 2 \mathrm{H}), 4.65$ (br s, 1H), 4.74 (br s, 1H), 6.35 (dd, 1H, $J=1.7,5.9 \mathrm{~Hz}), 6.37$ (dd, 1H, $J=1.5,5.9 \mathrm{~Hz}), 7.25-7.47$ (m, 6H), 7.65-7.69 (m, 4H); ${ }^{13} \mathrm{C}$ NMR (68 MHz) $\square$ 19.1, $26.8 \square 3,42.5,42.7,62.6,64.4,80.8,81.2,127.8 \square 4$, 129.9 2, $132.9 \square 2,135.5 \square$ 4, 135.6, 135.8; HRMS calcd for $\mathrm{C}_{20} \mathrm{H}_{21} \mathrm{O}_{3} \mathrm{Si}\left(\mathrm{M}^{+}-t-\mathrm{C}_{4} \mathrm{H}_{9}\right) m / z$ 337.1260, found 337.1258.

\section{(1S,2S,3R,4R)-2-Acetoxymethyl-3-[(1RS)-1-ethoxyethoxy]methyl-7-oxabicyclo[2.2.1]hept-5-}

ene ((+)-11). To a stirred solution of (+)-10 (2.04 g, $10.3 \mathrm{mmol})$ in $\mathrm{CH}_{2} \mathrm{Cl}_{2}(40 \mathrm{~mL})$ were added ethyl vinyl ether $(1.5 \mathrm{~mL}, 16 \mathrm{mmol})$ and PPTS $(265 \mathrm{mg}, 1.06 \mathrm{mmol})$. The mixture was stirred for $1 \mathrm{~h}$, diluted with saturated aqueous $\mathrm{NaHCO}_{3}(200 \mathrm{~mL})$, and extracted with $\mathrm{CH}_{2} \mathrm{Cl}_{2}(100 \mathrm{~mL} \square$ 4). The combined extracts were dried and concentrated in vacuo. The residue was purified by column chromatography on silica gel (EtOAc:hexane, 1:5 to 1:2) to provide $2.71 \mathrm{~g}(97 \%)$ of $(+)-\mathbf{1 1}($ d.r. $=c a .1: 1)$ as a pale yellow oil: TLC $R_{f} 0.80$ (EtOAc:hexane, 1:1); [ $]_{\mathrm{D}}{ }^{27}+7.3\left(c 3.69, \mathrm{CHCl}_{3}\right)$; IR 2940, $1740 \mathrm{~cm}^{-1} ;{ }^{1} \mathrm{H}$ NMR $(270 \mathrm{MHz})$

\footnotetext{
${ }^{3}$ The 4-t-butylbenzoate sample was prepared by treatment of (+)-1 $\mathbf{0}$ or (-)-1 $\mathbf{0}$ with $t$-BuBzCl in pyridine.
} 
$\square 1.21(\mathrm{t}, 3 \mathrm{H}, J=7.2 \mathrm{~Hz}), 1.32(\mathrm{~d}, 3 \mathrm{H} \square 1 / 2, J=5.1 \mathrm{~Hz}), 1.34(\mathrm{~d}, 3 \mathrm{H} \square 1 / 2, J=5.5 \mathrm{~Hz}), 1.93-1.98(\mathrm{~m}, 2 \mathrm{H})$, 2.09 (s, 3H), 3.35 (m, $1 \mathrm{H} \square$ 1/2), 3.50 (q, 2H $\square 1 / 2, J=7.2 \mathrm{~Hz}$ ), 3.51 (q, $2 \mathrm{H} \square$ 1/2, J = 7.2 Hz), 3.61-3.75 $(\mathrm{m}, 1 \mathrm{H} \square 1 / 2+1 \mathrm{H}), 3.95(\mathrm{~m}, 1 \mathrm{H}), 4.30(\mathrm{~m}, 1 \mathrm{H}), 4.70(\mathrm{q}, 1 \mathrm{H} \square 1 / 2, J=5.1 \mathrm{~Hz}), 4.71(\mathrm{q}, 1 \mathrm{H} \square 1 / 2, J=5.5$ $\mathrm{Hz}$ ), 4.81 (br s, 1H), 4.85 (br s, $1 \mathrm{H} \square 1 / 2$ ), 4.87 (br s, $1 \mathrm{H} \square 1 / 2$ ), 6.36 (dd, 1H, J=1.5, 5.9 Hz), 6.40 (dd, $1 \mathrm{H}, J=1.8,5.9 \mathrm{~Hz}) ;{ }^{13} \mathrm{C}$ NMR $(68 \mathrm{MHz}) \square 15.3,19.78 \square 1 / 2,19.84 \square 1 / 2,21.0,38.9,40.1,61.2,64.0 \square$ 1/2, $64.2 \square 1 / 2,64.5,80.4,80.7,99.8 \square 1 / 2,100.0 \square 1 / 2,135.1,135.9,170.9$; HRMS calcd for $\mathrm{C}_{13} \mathrm{H}_{19} \mathrm{O}_{5}$ $\left(\mathrm{M}^{+}-\mathrm{CH}_{3}\right) \mathrm{m} / \mathrm{z}$ 255.1233, found 255.1239.

\section{$(1 R, 2 R, 3 S, 4 S)-2-[(1 R S)-(1-E t h o x y e t h o x y)]$ methyl-3-hydroxymethyl-7-oxabicyclo[2.2.1]hept-5-} ene $((+)-12)$. To a stirred solution of $(+)-11(2.71 \mathrm{~g}, 10.0 \mathrm{mmol})$ in $\mathrm{MeOH}(50 \mathrm{~mL})$ was added $\mathrm{NaOMe}$ (1.0 M solution in $\mathrm{MeOH}, 2.0 \mathrm{~mL}, 2.0 \mathrm{mmol})$. The mixture was stirred for $2 \mathrm{~h}$, diluted with saturated brine $(250 \mathrm{~mL})$, and extracted with EtOAc $(100 \mathrm{~mL} \square$ 4). The combined extracts were dried and concentrated in vacuo. The residue was purified by column chromatography on silica gel (EtOAc:hexane, 1:3) to provide $2.30 \mathrm{~g}$ (quant.) of $(+)-\mathbf{1 2}$ (d.r. $=c a .1: 1)$ as a colorless oil: TLC $R_{f} 0.29$ (EtOAc:hexane, 1:1); $[\square]_{\mathrm{D}}^{22}+9.8\left(c 0.710, \mathrm{CHCl}_{3}\right)$; IR 3440, $2940 \mathrm{~cm}^{-1} ;{ }^{1} \mathrm{H} \mathrm{NMR}(270 \mathrm{MHz}) \square 1.22(\mathrm{t}, 3 \mathrm{H}, J=7.0 \mathrm{~Hz})$, $1.34(\mathrm{~d}, 3 \mathrm{H} \square 1 / 2, J=5.1 \mathrm{~Hz}), 1.35(\mathrm{~d}, 3 \mathrm{H} \square 1 / 2, J=5.1 \mathrm{~Hz}), 1.89-2.04(\mathrm{~m}, 2 \mathrm{H}), 2.83(\mathrm{br}, 1 \mathrm{H} \square 1 / 2, \mathrm{OH})$, 2.95 (br, $1 \mathrm{H} \mathrm{1} 1 / 2, \mathrm{OH}), 3.45-3.88(\mathrm{~m}, 6 \mathrm{H}), 4.69-4.77(\mathrm{~m}, 3 \mathrm{H}), 6.37-6.43(\mathrm{~m}, 2 \mathrm{H}) ;{ }^{13} \mathrm{C} \mathrm{NMR}(68 \mathrm{MHz}) \square$ 15.3, 19.7, $40.1 \square 1 / 2,40.3 \square 1 / 2,42.7 \square 1 / 2,42.8 \square 1 / 2,61.1 \square 1 / 2,61.4 \square 1 / 2,62.5,65.1 \square 1 / 2,65.5 \square$ $1 / 2,81.1 \square 1 / 2,81.2 \square 1 / 2,81.3,99.9 \square 1 / 2,100.0 \square 1 / 2,135.5,135.9$; HRMS calcd for $\mathrm{C}_{10} \mathrm{H}_{15} \mathrm{O}_{3}$ $\left(\mathrm{M}^{+}-\mathrm{OC}_{2} \mathrm{H}_{5}\right) \mathrm{m} / \mathrm{z}$ 183.1021, found 183.1016.

\section{$(1 S, 2 S, 3 R, 4 R)-2-(t$-Butyldiphenylsilyloxy)methyl-3-[(1RS)-1-ethoxyethoxy]methyl-7-oxa-}

bicyclo[2.2.1] hept-5-ene $((-)-13)$. To a cooled $\left(0^{\circ} \mathrm{C}\right)$ stirred solution of $(+)-12(2.30 \mathrm{~g}, 10.0 \mathrm{mmol})$ in DMF (40 mL) were added imidazole $(1.50 \mathrm{~g}, 22.0 \mathrm{mmol})$ and TBDPSCl $(2.9 \mathrm{~mL}, 11 \mathrm{mmol})$. The mixture was sitrred for $1 \mathrm{~h}$, diluted with EtOAc $(200 \mathrm{~mL})$, and washed with $\mathrm{H}_{2} \mathrm{O}(100 \mathrm{~mL} \square 3)$. The organic layer was dried and concentrated in vacuo to provide $6.12 \mathrm{~g}$ of crude $(-)-\mathbf{1 3}$ (d.r. $=c a .1: 1$ ), which was used directly in the next step. For the one-pot ROM/CM/RCM reaction, crude (-)-13 was purified by column chromatography on silica gel (EtOAc:hexane, 1:5) and obtained in quantitative yield as a colorless oil: TLC

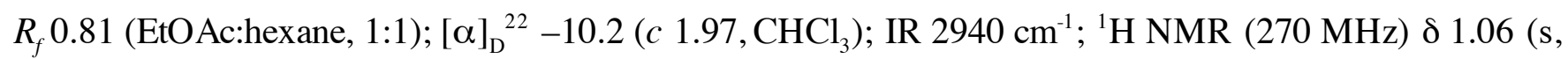
$9 \mathrm{H}), 1.12(\mathrm{t}, 3 \mathrm{H} \square 1 / 2, J=7.1 \mathrm{~Hz}), 1.15(\mathrm{t}, 3 \mathrm{H} \square 1 / 2, J=7.1 \mathrm{~Hz}), 1.20(\mathrm{~d}, 3 \mathrm{H} \square 1 / 2, J=5.4 \mathrm{~Hz}), 1.24(\mathrm{~d}$, $3 \mathrm{H} \square 1 / 2, J=5.3 \mathrm{~Hz}), 1.83-1.97(\mathrm{~m}, 2 \mathrm{H}), 3.21-3.81(\mathrm{~m}, 6 \mathrm{H}), 4.57(\mathrm{q}, 1 \mathrm{H} \square 1 / 2, J=5.4 \mathrm{~Hz}), 4.60(\mathrm{q}, 1 \mathrm{H} \square$ $1 / 2, J=5.3 \mathrm{~Hz}$ ), 4.82 (br s, $1 \mathrm{H}$ ), 4.93 (br s, $1 \mathrm{H} \square 1 / 2$ ), 4.94 (br s, $1 \mathrm{H} \square 1 / 2$ ), 6.36 (br s, 2H), 7.38-7.43 (m, 6H), 7.64-7.68 (m, 4H); ${ }^{13} \mathrm{C}$ NMR (68 MHz) $\square 15.2,19.2,19.8,26.8 \square 3,40.0,42.4,60.9 \square 1 / 2,61.0 \square 1 / 2$, 63.3, $64.5 \square 1 / 2,64.8 \square 1 / 2,80.3,80.7,99.7 \square 1 / 2,100.0 \square 1 / 2,127.7 \square 4,129.7 \square 2,133.6,133.7,135.5 \square$ 6; HRMS calcd for $\mathrm{C}_{24} \mathrm{H}_{29} \mathrm{O}_{4} \mathrm{Si}\left(\mathrm{M}^{+}-t-\mathrm{C}_{4} \mathrm{H}_{9}\right) \mathrm{m} / z$ 409.1835, found 409.1830.

\section{$(1 S, 2 S, 3 R, 4 R)-2-(t$-Butyldiphenylsilyloxy)methyl-3-hydroxymethyl-7-oxabicyclo[2.2.1]hept-5-}

ene ((-) -7). A solution of crude (-)-13 obtained above $(6.12 \mathrm{~g})$ in THF $(40 \mathrm{~mL})$ and $60 \%$ aqueous $\mathrm{AcOH}(120 \mathrm{~mL})$ was stirred for $5 \mathrm{~h}$ and concentrated in vacuo with aid of EtOH. The residue was purified by column chromatography on silica gel (EtOAc:hexane, 1:2) to provide $3.82 \mathrm{~g}$ (97\% from (+)12) of (-)-7 as white crystals: $\mathrm{mp} 65-67^{\circ} \mathrm{C}$; $[\square]_{\mathrm{D}}{ }^{22}-14.0\left(c 1.46, \mathrm{CHCl}_{3}\right)$. 
exo-cis-2,3-Bis(acetoxymethyl) -7-oxabicyclo[2.2.1] hept-5-ene (14). To a cooled $\left(0{ }^{\circ} \mathrm{C}\right)$ stirred solution of $\mathbf{8}^{4}(3.01 \mathrm{~g}, 18.1 \mathrm{mmol})$ in THF $(60 \mathrm{~mL})$ was added $\mathrm{LiAlH}_{4}(1.36 \mathrm{~g}, 36.8 \mathrm{mmol})$ in four portions over a period of $2 \mathrm{~h}$. The mixture was stirred for $20 \mathrm{~h}$ and quenched with $\mathrm{H}_{2} \mathrm{O}(1.4 \mathrm{~mL}), 10 \mathrm{wt} \%$ aqueous $\mathrm{NaOH}(1.4 \mathrm{~mL})$, and $\mathrm{H}_{2} \mathrm{O}(4.2 \mathrm{~mL})$, successively. The precipitated solids were removed by filtration through a Celite-pad and washed well with EtOAc. The combined filtrate and washings were concentrated in vacuo to provide crude 9 (2.56 g), which was used directly in the next step.

A solution of crude $9(2.56 \mathrm{~g})$ in $\mathrm{Ac}_{2} \mathrm{O}(20 \mathrm{~mL})$ and pyridine $(20 \mathrm{~mL})$ was stirred for $17 \mathrm{~h}$ and concentrated in vacuo with aid of toluene. The residue was purified by column chromatography on silica gel (EtOAc:hexane, 1:3) to provide $2.92 \mathrm{~g}(67 \%)$ of $\mathbf{1 4}$ as white crystals: mp 101-102 ${ }^{\circ} \mathrm{C}$; TLC $R_{f} 0.57$ (EtOAc:hexane, 1:1); IR (KBr) 2940, $1740 \mathrm{~cm}^{-1}$; ${ }^{1} \mathrm{H}$ NMR (270 MHz) $\square$ 1.97-2.09 (m, 2H), 2.09 (s, 6H), $4.02(\mathrm{dd}, 2 \mathrm{H}, J=9.5,10.9 \mathrm{~Hz}), 4.29(\mathrm{dd}, 2 \mathrm{H}, J=5.3,10.9 \mathrm{~Hz}), 4.82(\mathrm{~s}, 2 \mathrm{H}), 6.40(\mathrm{~s}, 2 \mathrm{H}) ;{ }^{3} \mathrm{C}$ NMR $(68$ $\mathrm{MHz}) \square 21.0 \square 2,39.2 \square 2,63.8 \square 2,80.5 \square 2,135.6 \square 2,170.8 \square 2$; HRMS calcd for $\mathrm{C}_{12} \mathrm{H}_{17} \mathrm{O}_{5}\left(\mathrm{M}^{+}+\mathrm{H}\right)$ $\mathrm{m} / \mathrm{z}$ 241.1076, found 241.1076.

$(1 R, 2 R, 3 S, 4 S)$-2-Acetoxymethyl-3-hydroxymethyl-7-oxabicyclo[2.2.1] hept-5-ene ((-)-10). To a stirred solution of $14(1.48 \mathrm{~g}, 6.15 \mathrm{mmol})$ in $\mathrm{H}_{2} \mathrm{O}(60 \mathrm{~mL})$ and THF $(60 \mathrm{~mL})$ was added lipase PS (Amano) $(591 \mathrm{mg})$. The mixture was stirred for 20 days, diluted with saturated brine $(300 \mathrm{~mL})$, and extracted with EtOAc (50 mL $\square$ ). The combined extracts were dried and concentrated in vacuo. The residue was purified by column chromatography on silica gel (EtOAc:hexane, 1:2) to provide $1.07 \mathrm{~g}(88 \%)$ of (-)-10 and $93 \mathrm{mg}(6.3 \%)$ of $\mathbf{1 4}$ was recovered. Compound (-)-10 was obtained as white crystals: $\mathrm{mp}$ 49-51 ${ }^{\circ} \mathrm{C} ;[\square]_{\mathrm{D}}{ }^{24}-7.7(c 1.48, \mathrm{MeOH})$. As described for the HPLC analysis of (+)-10, compound (-)-10 was determined to be $95 \%$ ee.

Preparation of (-) -7 from (-) -10. As described for the preparation of (+)-7 from (+)-10, compound (-)-10 (1.02 g, $5.12 \mathrm{mmol})$ was converted to $1.90 \mathrm{~g}(94 \%)$ of (-)-7.

\section{(1S,2S,3R,4R)-2-(t-Butyldiphenylsilyloxy)methyl-3-(methyldithiocarbonyloxy)methyl-7-oxa-}

bicyclo[2.2.1] hept-5-ene ((-) -15). To a cooled $\left(0^{\circ} \mathrm{C}\right)$ stirred solution of $(-)-7(3.67 \mathrm{~g}, 9.30 \mathrm{mmol})$ in THF $(75 \mathrm{~mL})$ was added $\mathrm{NaH}(\mathrm{ca} .60 \%$ in oil, $0.558 \mathrm{~g}, 14.0 \mathrm{mmol})$. After being stirred for $30 \mathrm{~min}$, the mixture was cooled to $0{ }^{\circ} \mathrm{C}$, and $\mathrm{CS}_{2}(1.12 \mathrm{~mL}, 18.6 \mathrm{mmol})$ and $\mathrm{MeI}(1.15 \mathrm{~mL}, 18.6 \mathrm{mmol})$ were added successively. The mixture was stirred for $2 \mathrm{~h}$, quenched with $\mathrm{H}_{2} \mathrm{O}(2 \mathrm{~mL})$, diluted with saturated brine (200 mL), and extracted with $\mathrm{CH}_{2} \mathrm{Cl}_{2}(100 \mathrm{~mL} \mathrm{3})$. The combined extracts were dried and concentrated in vacuo. The residue was purified by column chromatography on silica gel (EtOAc:hexane, 1:20) to provide $4.33 \mathrm{~g}(96 \%)$ of (-)-15 as a pale yellow oil: TLC $R_{f} 0.57$ (EtOAc:hexane, 1:3); [ $]_{\mathrm{D}}{ }^{23}-8.7$ (c 1.70, $\mathrm{CHCl}_{3}$ ); IR $2940 \mathrm{~cm}^{-1}$; ${ }^{1} \mathrm{H}$ NMR (270 MHz) $\square 1.06$ (s, 9H), 1.99 (dt, 1H, J= 7.1, 8.4 Hz), 2.15 (ddd, 1H, $J$ $=5.1,8.4,10.6 \mathrm{~Hz}), 2.52(\mathrm{~s}, 3 \mathrm{H}), 3.70(\mathrm{dd}, 1 \mathrm{H}, J=8.4,9.9 \mathrm{~Hz}), 3.78(\mathrm{dd}, 1 \mathrm{H}, J=7.1,9.9 \mathrm{~Hz}), 4.49(\mathrm{t}, 1 \mathrm{H}$, $J=10.6 \mathrm{~Hz}$ ), 4.84 (br s, 1H), 4.86 (br s, 1H), 4.94 (dd, 1H, $J=5.1,10.6 \mathrm{~Hz}), 6.38$ (dd, $1 \mathrm{H}, J=1.5,5.9$ $\mathrm{Hz}), 6.40(\mathrm{dd}, 1 \mathrm{H}, J=1.5,5.9 \mathrm{~Hz}), 7.35-7.44(\mathrm{~m}, 6 \mathrm{H}), 7.65-7.69(\mathrm{~m}, 4 \mathrm{H}) ;{ }^{13} \mathrm{C} \mathrm{NMR}(68 \mathrm{MHz}) \square 19.1 \square 2$, $26.8 \square 3,38.8,42.7,63.3,73.8,80.3,80.5,127.7 \square 4,129.7 \square 2,133.3 \square 2,135.1,135.5 \square 4,136.1,215.8$; HRMS calcd for $\mathrm{C}_{22} \mathrm{H}_{23} \mathrm{O}_{3} \mathrm{~S}_{2} \mathrm{Si}\left(\mathrm{M}^{+}-t-\mathrm{C}_{4} \mathrm{H}_{9}\right) \mathrm{m} / z$ 427.0858, found 427.0859.

\footnotetext{
${ }^{4}$ (a) Woodward, R. B.; Baer, H. J. Am. Chem. Soc. 1948, 70, 1161-1166. (b) Chem. Abstr. 1966, 65, 16924e.
} 


\section{$(1 R, 2 S, 3 R, 4 S, 5 R, 6 S)-2-(t$-Butyldiphenylsilyloxy)methyl-5,6-dihydroxy-3-(methyldithio-}

carbonyloxy) methyl-7-oxabicyclo[2.2.1] heptane ((-)-16). To a cooled $\left(0{ }^{\circ} \mathrm{C}\right)$ stirred solution of $(-)$ $15(3.03 \mathrm{~g}, 6.25 \mathrm{mmol})$ in $80 \%$ aqueous acetone $(60 \mathrm{~mL})$ were added $\mathrm{OsO}_{4}(0.05 \mathrm{M}$ solution in $t$ - $\mathrm{BuOH}$, $6.3 \mathrm{~mL}, 0.31 \mathrm{mmol})$ and $\mathrm{Me}_{3} \mathrm{NO} \cdot 2 \mathrm{H}_{2} \mathrm{O}(2.34 \mathrm{~g}, 31.3 \mathrm{mmol})$. The mixture was stirred for $20 \mathrm{~h}$, quenched with $1 \mathrm{M}$ aqueous $\mathrm{NaHSO}_{3}(1 \mathrm{~mL})$, diluted with EtOAc $(300 \mathrm{~mL})$, and washed with $1 \mathrm{M}$ aqueous $\mathrm{NaHSO}_{3}$ $(150 \mathrm{~mL} \square 2)$. The organic layer was dried and concentrated in vacuo. The residue was purified by column chromatography on silica gel (EtOAc:hexane, 1:2) to provide $2.76 \mathrm{~g}(85 \%)$ of (-)-16 as a colorless oil: TLC $R_{f} 0.06$ (EtOAc:hexane, 1:3); [ $]_{\mathrm{D}}{ }^{24}-17.0\left(c\right.$ 1.87, $\mathrm{CHCl}_{3}$ ); IR 3360, $2940 \mathrm{~cm}^{-1}$; ${ }^{1} \mathrm{H}$ NMR (270 $\mathrm{MHz}) \square 1.04$ (s, 9H), 2.04 (m, 1H), 2.28 (m, 1H), 2.50 (s, 3H), 3.13 (br, 2H, OH $\square$ 2), 3.62 (d, 2H, J= 7.7 $\mathrm{Hz}), 3.87(\mathrm{~d}, 1 \mathrm{H}, J=6.0 \mathrm{~Hz}), 3.91(\mathrm{~d}, 1 \mathrm{H}, J=6.0 \mathrm{~Hz}), 4.21(\mathrm{~d}, 1 \mathrm{H}, J=1.1 \mathrm{~Hz}), 4.26(\mathrm{~d}, 1 \mathrm{H}, J=1.1 \mathrm{~Hz})$, $4.43(\mathrm{t}, 1 \mathrm{H}, J=11.0 \mathrm{~Hz}), 4.83(\mathrm{dd}, 1 \mathrm{H}, J=5.5,11.0 \mathrm{~Hz}), 7.35-7.44(\mathrm{~m}, 6 \mathrm{H}), 7.65-7.69(\mathrm{~m}, 4 \mathrm{H}) ;{ }^{13} \mathrm{C} \mathrm{NMR}$ $(68 \mathrm{MHz}) \square 19.1 \square 2,26.8 \square 3,39.8,43.3,61.8,71.7,74.1,74.3,84.3,84.6,127.8 \square 4,129.8 \square 2,133.0 \square$ 2, $135.5 \square$ 4, 215.5; HRMS calcd for $\mathrm{C}_{22} \mathrm{H}_{25} \mathrm{O}_{5} \mathrm{~S}_{2} \mathrm{Si}\left(\mathrm{M}^{+}-t-\mathrm{C}_{4} \mathrm{H}_{9}\right) \mathrm{m} / z$ 461.0913, found 461.0903 .

\section{$(1 R, 2 S, 3 S, 4 S, 5 R, 6 S)$-2-(t-Butyldiphenylsilyloxy)methyl-5,6-dihydroxy-3-methyl-7-oxabicyclo-}

[2.2.1] heptane $((+)-17)$. The following reaction was carried out under Ar. To a refluxing solution of $(-)-16(2.76 \mathrm{~g}, 5.32 \mathrm{mmol})$ in toluene $(50 \mathrm{~mL})$ was added dropwise a solution of AIBN (262 $\mathrm{mg}, 1.60$ $\mathrm{mmol})$ and $n-\mathrm{Bu}_{3} \mathrm{SnH}(2.9 \mathrm{~mL}, 11 \mathrm{mmol})$ in toluene $(50 \mathrm{~mL})$ over $30 \mathrm{~min}$. The mixture was refluxed for an additional $1 \mathrm{~h}$ and concentrated in vacuo. The residue was purified by column chromatography on silica gel (EtOAc:hexane, 1:10 to 1:1) to provide $1.94 \mathrm{~g}(89 \%)$ of (+)-17 as a colorless oil: TLC $R_{f} 0.14$

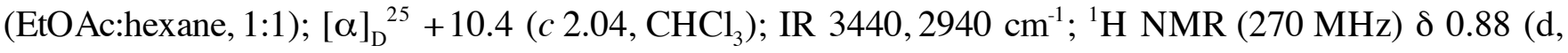
$3 \mathrm{H}, J=6.6 \mathrm{~Hz}), 1.05(\mathrm{~s}, 9 \mathrm{H}), 1.82-1.88(\mathrm{~m}, 2 \mathrm{H}), 3.31$ (br, 2H, OH $\square 2), 3.59$ (d, 2H, J = 6.2 Hz), 3.85 (br, $2 \mathrm{H}), 3.86(\mathrm{~d}, 1 \mathrm{H}, J=1.5 \mathrm{~Hz}), 4.31(\mathrm{~d}, 1 \mathrm{H}, J=1.8 \mathrm{~Hz}), 7.35-7.46(\mathrm{~m}, 6 \mathrm{H}), 7.63-7.67(\mathrm{~m}, 4 \mathrm{H}) ;{ }^{13} \mathrm{C}$ NMR (68 MHz) $\square 13.3,19.1,26.7 \square 3,34.4,43.4,62.1,74.1,74.3,84.4,88.8,127.5 \square 4,129.5 \square 2,133.3,133.4$, $135.3 \square 4$; HRMS calcd for $\mathrm{C}_{20} \mathrm{H}_{23} \mathrm{O}_{4} \mathrm{Si}\left(\mathrm{M}^{+}-t-\mathrm{C}_{4} \mathrm{H}_{9}\right) \mathrm{m} / z$ 355.1366, found 355.1368.

\section{$(2 R, 3 S, 4 S, 5 S)-3-(t$-Butyldiphenylsilyloxy)methyl-2,5-bis(hydroxymethyl)-4-methyl-}

tetrahydrofuran $((+)-18)$. To a stirred solution of $(+)-17(2.51 \mathrm{~g}, 6.09 \mathrm{mmol})$ in benzene $(50 \mathrm{~mL})$ was added $\mathrm{Pb}(\mathrm{OAc})_{4}(4.05 \mathrm{~g}, 9.14 \mathrm{mmol})$. The mixture was stirred for $45 \mathrm{~min}$, and the precipitated solids were removed by filtration through a Celite-pad and washed well with EtOAc. The combined filtrate and washings were concentrated in vacuo to provide crude dialdehyde monohydrate $(3.85 \mathrm{~g})$, which was used immediately in the next step.

To a cooled $\left(0{ }^{\circ} \mathrm{C}\right)$ stirred solution of crude dialdehyde monohydrate $(3.85 \mathrm{~g})$ in $\mathrm{MeOH}(70 \mathrm{~mL})$ was added $\mathrm{NaBH}_{4}(2.3 \mathrm{~g}, 61 \mathrm{mmol})$. After being stirred for $1 \mathrm{~h}$, the mixture was cooled to $0{ }^{\circ} \mathrm{C}$, and $35 \%$ aqueous $\mathrm{H}_{2} \mathrm{O}_{2}(4 \mathrm{~mL})$ was added. The mixture was stirred for $1 \mathrm{~h}$, quenched with saturated aqueous $\mathrm{Na}_{2} \mathrm{SO}_{3}(15 \mathrm{~mL})$ at $0{ }^{\circ} \mathrm{C}$, diluted with saturated brine $(200 \mathrm{~mL})$, and extracted with $\mathrm{CH}_{2} \mathrm{Cl}_{2}(200 \mathrm{~mL} \square)$. The combined extracts were dried and concentrated in vacuo. The residue was purified by column chromatography on silica gel (EtOAc:hexane, 1:1) to provide $2.04 \mathrm{~g}(81 \%)$ of $(+)-\mathbf{1 8}$ as a colorless oil:

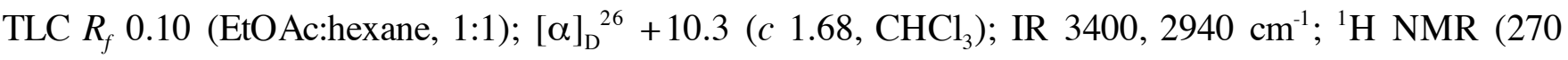
$\mathrm{MHz}) \square 0.93(\mathrm{~d}, 3 \mathrm{H}, J=6.8 \mathrm{~Hz}), 1.05$ (s, 9H), 2.21-2.32 (m, 4H), 3.53 (dd, 1H, J = 4.4, $11.7 \mathrm{~Hz}), 3.59$ (dd, 
$1 \mathrm{H}, J=4.9,11.7 \mathrm{~Hz}), 3.64-3.75(\mathrm{~m}, 3 \mathrm{H}), 3.79(\mathrm{dd}, 1 \mathrm{H}, J=3.4,11.7 \mathrm{~Hz}), 3.80(\mathrm{dd}, 1 \mathrm{H}, J=2.7,11.7 \mathrm{~Hz})$, $4.07(\mathrm{~m}, 1 \mathrm{H}), 7.36-7.45(\mathrm{~m}, 6 \mathrm{H}), 7.64-7.67(\mathrm{~m}, 4 \mathrm{H}) ;{ }^{13} \mathrm{C} \mathrm{NMR}(68 \mathrm{MHz}) \square 12.3,19.1,26.8 \mathrm{~B}, 35.8,45.4$, 62.7, 63.1, 65.1, 82.4, 86.2, $127.7 \square 4,129.7 \square 2,133.1,133.2,135.5 \square 4$; HRMS calcd for $\mathrm{C}_{20} \mathrm{H}_{25} \mathrm{O}_{4} \mathrm{Si}$ $\left(\mathrm{M}^{+}-t-\mathrm{C}_{4} \mathrm{H}_{9}\right) m / z 357.1522$, found 357.1519 .

\section{$(2 R, 3 S, 4 S, 5 S)$-3-( $t$-Butyldiphenylsilyloxy)methyl-4-methyl-2,5-bis[(p-toluenesulfonyloxy)-} methyl] tetrahydrofuran $((+)-19)$. The following reaction was carried out under Ar. To a cooled $\left(0{ }^{\circ} \mathrm{C}\right)$ stirred solution of $(+)-\mathbf{1 8}(2.04 \mathrm{~g}, 4.92 \mathrm{mmol})$ in THF $(100 \mathrm{~mL})$ was added $n$-BuLi $(2.44 \mathrm{M}$ solution in hexane, $5.0 \mathrm{~mL}, 12 \mathrm{mmol})$. The mixture was stirred for $1 \mathrm{~h}$ and $\mathrm{TsCl}(2.35 \mathrm{~g}, 12.3 \mathrm{mmol})$ was added at $0{ }^{\circ} \mathrm{C}$. After being stirred for $30 \mathrm{~min}$, the mixture was diluted with saturated aqueous $\mathrm{NH}_{4} \mathrm{Cl}(250$ $\mathrm{mL})$ and extracted with $\mathrm{CH}_{2} \mathrm{Cl}_{2}(120 \mathrm{~mL} \quad 3)$. The combined extracts were dried and concentrated in vacuo. The residue was purified by column chromatography on silica gel (EtOAc:hexane, 1:5) to provide $2.82 \mathrm{~g}(79 \%)$ of $(+)-19$ as a pale yellow oil: TLC $R_{f} 0.68$ (EtOAc:hexane, 1:1); [0] $]_{\mathrm{D}}{ }^{26}+6.3(c 1.42$, $\mathrm{CHCl}_{3}$ ); IR $2940 \mathrm{~cm}^{-1}$; ${ }^{1} \mathrm{H}$ NMR (270 MHz) $\square 0.86$ (d, 3H, $\left.J=6.6 \mathrm{~Hz}\right), 0.99$ (s, 9H), 2.05-2.20 (m, 2H), 2.43 (s, 3H), 2.44 (s, 3H), 3.56 (dd, $1 \mathrm{H}, J=6.2,10.3 \mathrm{~Hz}), 3.63(\mathrm{dd}, 1 \mathrm{H}, J=4.8,10.3 \mathrm{~Hz}), 3.73(\mathrm{~m}, 1 \mathrm{H})$, $3.89(\mathrm{dd}, 1 \mathrm{H}, J=4.7,10.3 \mathrm{~Hz}), 3.91(\mathrm{dd}, 1 \mathrm{H}, J=4.7,9.9 \mathrm{~Hz}), 4.00(\mathrm{dd}, 1 \mathrm{H}, J=4.4,10.3 \mathrm{~Hz}), 4.03(\mathrm{dd}$, $1 \mathrm{H}, J=3.7,9.9 \mathrm{~Hz}), 4.10(\mathrm{~m}, 1 \mathrm{H}), 7.29-7.45(\mathrm{~m}, 10 \mathrm{H}), 7.58-7.61(\mathrm{~m}, 4 \mathrm{H}), 7.74-7.81(\mathrm{~m}, 4 \mathrm{H}) ;{ }^{13} \mathrm{C} \mathrm{NMR}$ (68 MHz) $\square$ 12.1, 19.1, $21.7 \square$ 2, $26.8 \square$ 3, 36.5, 45.3, 62.1, 70.1, 71.3, 79.1, 82.9, $127.8 \square$ 4, $127.9 \square 4$, $129.9 \square 6,132.7 \square 2,132.8,133.0,135.5 \square 4,144.8,144.9 ;$ HRMS calcd for $\mathrm{C}_{34} \mathrm{H}_{37} \mathrm{O}_{8} \mathrm{~S}_{2} \mathrm{Si}\left(\mathrm{M}^{+}-t-\mathrm{C}_{4} \mathrm{H}_{9}\right)$ $\mathrm{m} / \mathrm{z} 665.1699$, found 665.1695 .

\section{( $2 R, 3 S, 4 S, 5 S)$-3-(t-Butyldiphenylsilyloxy)methyl-2,5-bis(iodomethyl)-4-methyltetrahydro-}

furan $((+)-20)$. To a stirred solution of $(+)-19(2.82 \mathrm{~g}, 3.90 \mathrm{mmol})$ in 2-butanone $(60 \mathrm{~mL})$ was added $\mathrm{NaI}(2.05 \mathrm{~g}, 13.7 \mathrm{mmol})$. The mixture was refluxed for $12 \mathrm{~h}$, diluted with EtOAc $(220 \mathrm{~mL})$, and washed with saturated aqueous $\mathrm{Na}_{2} \mathrm{~S}_{2} \mathrm{O}_{3}(70 \mathrm{~mL} \square 2)$ and saturated brine $(70 \mathrm{~mL})$. The organic layer was dried and concentrated in vacuo. The residue was purified by column chromatography on silica gel (EtOAc:hexane, 1:20) to provide $2.36 \mathrm{~g}(96 \%)$ of (+)-20 as a colorless oil: TLC $R_{f} 0.71$ (EtOAc:hexane, 1:3); []$_{\mathrm{D}}{ }^{27}+0.5\left(c 1.81, \mathrm{CHCl}_{3}\right)$; IR $2940 \mathrm{~cm}^{-1} ;{ }^{1} \mathrm{H} \mathrm{NMR}(270 \mathrm{MHz}) \square 0.96(\mathrm{~d}, 3 \mathrm{H}, J=6.6 \mathrm{~Hz}), 1.06$ (s, $9 \mathrm{H}), 2.19-2.33(\mathrm{~m}, 2 \mathrm{H}), 3.23(\mathrm{dd}, 1 \mathrm{H}, J=5.1,10.6 \mathrm{~Hz}), 3.30(\mathrm{dd}, 1 \mathrm{H}, J=4.8,10.3 \mathrm{~Hz}), 3.38(\mathrm{dd}, 1 \mathrm{H}, J=$ $5.1,10.6 \mathrm{~Hz}), 3.39(\mathrm{dd}, 1 \mathrm{H}, J=4.8,10.3 \mathrm{~Hz}), 3.59(\mathrm{dt}, 1 \mathrm{H}, J=6.6,5.1 \mathrm{~Hz}), 3.67(\mathrm{dd}, 1 \mathrm{H}, J=6.6,10.4 \mathrm{~Hz})$, $3.72(\mathrm{dd}, 1 \mathrm{H}, J=5.1,10.4 \mathrm{~Hz}), 3.96(\mathrm{dt}, 1 \mathrm{H}, J=5.5,4.8 \mathrm{~Hz}), 7.37-7.48(\mathrm{~m}, 6 \mathrm{H}), 7.64-7.68(\mathrm{~m}, 4 \mathrm{H}) ;{ }^{13} \mathrm{C}$ $\operatorname{NMR}(68 \mathrm{MHz}) \square 10.0,11.6,12.6,19.1,26.8 \square 3,40.5,49.1,62.5,80.8,84.7,127.8 \square$ 4, $129.8 \square 2,132.0$ प2, 135.6 4; HRMS calcd for $\mathrm{C}_{20} \mathrm{H}_{23} \mathrm{I}_{2} \mathrm{O}_{2} \mathrm{Si}\left(\mathrm{M}^{+}-t-\mathrm{C}_{4} \mathrm{H}_{9}\right) \mathrm{m} / z$ 576.9557, found 576.9558 .

\section{(2S,3S,4S,5R)-3-(t-Butyldiphenylsilyloxy)methyl-4-methyl-2,5-di(2-propenyl)tetrahydrofuran} $((+)-6)$. The following reaction was carried out under Ar. To a stirred solution of (+)-20 (2.00 g, 3.15 $\mathrm{mmol})$ in benzene $(80 \mathrm{~mL})$ was added vinyl $\mathrm{MgBr}(1.0 \mathrm{M}$ solution in THF, $25 \mathrm{~mL}, 25 \mathrm{mmol})$. The mixture was stirred for $20 \mathrm{~h}$, diluted with saturated aqueous $\mathrm{NH}_{4} \mathrm{Cl}(200 \mathrm{~mL})$, and extracted with EtOAc $(100 \mathrm{~mL} \backslash 2)$. The combined extracts were dried and concentrated in vacuo. The residue was purified by column chromatography on silica gel (toluene:hexane, 1:2) to provide $1.22 \mathrm{~g} \mathrm{(89 \% )} \mathrm{of} \mathrm{(+)-6} \mathrm{as} \mathrm{a} \mathrm{colorless}$ oil: TLC $R_{f} 0.57$ (toluene:hexane, 3:1); [ర] ${ }_{\mathrm{D}}^{23}+5.7\left(c 0.830, \mathrm{CHCl}_{3}\right)$; IR 2940, $1640 \mathrm{~cm}^{-1} ;{ }^{1} \mathrm{H}$ NMR (270 
$\mathrm{MHz}) \square 0.93(\mathrm{~d}, 3 \mathrm{H}, J=7.0 \mathrm{~Hz}), 1.04(\mathrm{~s}, 9 \mathrm{H}), 1.92-2.39(\mathrm{~m}, 6 \mathrm{H}), 3.53$ (dt, $1 \mathrm{H}, J=5.1,7.0 \mathrm{~Hz}), 3.61$ (dd, $1 \mathrm{H}, J=6.6,10.3 \mathrm{~Hz}), 3.68(\mathrm{dd}, 1 \mathrm{H}, J=5.9,10.3 \mathrm{~Hz}), 3.89(\mathrm{dt}, 1 \mathrm{H}, J=5.5,5.9 \mathrm{~Hz}), 4.99-5.12(\mathrm{~m}, 4 \mathrm{H})$, 5.71-5.95 (m, 2H), 7.35-7.42 (m, 6H), 7.64-7.68 (m, 4H); ${ }^{13} \mathrm{C}$ NMR (68 MHz) $\square$ 12.2, 19.2, $26.8 \square 3,38.8$, 39.4, 40.4, 48.0, 62.7, 80.1, 84.3, 116.6, 116.8, $127.7 \square$ 4, $129.6 \square 2$, $133.5 \square$ 2, 135.1, 135.2, $135.6 \square 4$; HRMS calcd for $\mathrm{C}_{24} \mathrm{H}_{29} \mathrm{O}_{2} \mathrm{Si}\left(\mathrm{M}^{+}-t-\mathrm{C}_{4} \mathrm{H}_{9}\right) m / z$ 377.1937, found 377.1942.

$(1 R, 6 S, 7 S, 8 S)$-7-( $t$-Butyldiphenylsilyloxy) methyl-8-methyl-9-oxabicyclo[4.2.1] non-3-ene $((+)$ 5). The following reaction was carried out with bubbling Ar. To a stirred solution of (+)-6 (850 mg, $1.96 \mathrm{mmol})$ in benzene $(650 \mathrm{~mL})$ was added a solution of 2nd generation Grubbs catalyst 22 (25 mg, $0.029 \mathrm{mmol})$ in benzene $(2 \mathrm{~mL})$. The mixture was refluxed for $4 \mathrm{~h}$ and a solution of $22(25 \mathrm{mg}, 0.029$ mmol) in benzene $(2 \mathrm{~mL})$ was added. The mixture was refluxed for $2 \mathrm{~h}$ and then DMSO (0.21 $\mathrm{mL}, 2.9$ mmol) was added. ${ }^{5}$ After being refluxed for $16 \mathrm{~h}$, the mixture was concentrated in vacuo. The residue was purified by column chromatography on silica gel (EtOAc:hexane, 1:80) to provide $689 \mathrm{mg}(86 \%)$ of

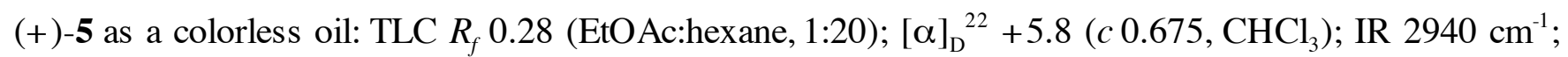
${ }^{1} \mathrm{H}$ NMR $(270 \mathrm{MHz}) \square 0.92(\mathrm{~d}, 3 \mathrm{H}, J=7.3 \mathrm{~Hz}), 1.04(\mathrm{~s}, 9 \mathrm{H}), 2.15-2.64(\mathrm{~m}, 6 \mathrm{H}), 3.59$ (dd, $1 \mathrm{H}, J=9.2,9.9$ $\mathrm{Hz}), 3.73(\mathrm{dd}, 1 \mathrm{H}, J=6.2,9.9 \mathrm{~Hz}), 3.98(\mathrm{~m}, 1 \mathrm{H}), 4.36(\mathrm{~m}, 1 \mathrm{H}), 5.50-5.63(\mathrm{~m}, 2 \mathrm{H}), 7.35-7.43(\mathrm{~m}, 6 \mathrm{H})$, 7.63-7.67 (m, 4H); ${ }^{13} \mathrm{C}$ NMR (68 MHz) $\square$ 15.4, 19.2, $26.8 \square 3,38.4,39.1,40.2,48.4,63.9,81.5,86.0$, 127.2, 127.5, $127.7 \square 4,129.6 \square 2,133.8 \square 2,135.5 \square 4$; HRMS calcd for $\mathrm{C}_{22} \mathrm{H}_{25} \mathrm{O}_{2} \mathrm{Si}\left(\mathrm{M}^{+}-t-\mathrm{C}_{4} \mathrm{H}_{9}\right) \mathrm{m} / z$ 349.1624 , found 349.1616 .

\section{(1R,3RS,4RS,6S,7S,8S)-3,4-Dibromo-7-(t-butyldiphenylsilyloxy)methyl-8-methyl-9-oxa-}

bicyclo[4.2.1]nonane (23). To a cooled $\left(-78^{\circ} \mathrm{C}\right)$ stirred solution of $(+)-5(780 \mathrm{mg}, 1.91 \mathrm{mmol})$ in $\mathrm{Et}_{2} \mathrm{O}$ $(30 \mathrm{~mL})$ was added a solution of bromine $(0.10 \mathrm{~mL}, 2.0 \mathrm{mmol})$ in $\mathrm{Et}_{2} \mathrm{O}(1 \mathrm{~mL})$. The mixture was stirred at $-78{ }^{\circ} \mathrm{C}$ for $1 \mathrm{~h}$, diluted with EtOAc $(100 \mathrm{~mL})$, and washed with saturated aqueous $\mathrm{Na}_{2} \mathrm{~S}_{2} \mathrm{O}_{3}(50 \mathrm{~mL}$ 2) and saturated brine $(50 \mathrm{~mL})$. The organic layer was dried and concentrated in vacuo. The residue was purified by column chromatography on silica gel (EtOAc:hexane, 1:60) to provide $1.03 \mathrm{~g}(96 \%)$ of 23 (d.r.= ca. 5:6) as a pale yellow oil: TLC $R_{f} 0.28$ (EtOAc:hexane, 1:20); IR $2940 \mathrm{~cm}^{-1} ;{ }^{1} \mathrm{H}$ NMR $(270 \mathrm{MHz})$ $\square 0.89(\mathrm{~d}, 3 \mathrm{H} \square 5 / 11, J=8.1 \mathrm{~Hz}), 0.91$ (d, 3H $\square$ 6/11, J= $7.0 \mathrm{~Hz}), 1.05$ (s, 9H $\square$ 6/11), 1.06 (s, 9H $\square 5 / 11)$, 1.76-1.87 (m, $1 \mathrm{H} \square 5 / 11), 2.06-2.26(\mathrm{~m}, 2 \mathrm{H}), 2.34-2.61(\mathrm{~m}, 2 \mathrm{H}+1 \mathrm{H} \square 6 / 11), 2.97-3.08(\mathrm{~m}, 1 \mathrm{H}), 3.54-$ $3.78(\mathrm{~m}, 2 \mathrm{H}), 3.83-4.12(\mathrm{~m}, 2 \mathrm{H}), 4.23-4.61(\mathrm{~m}, 2 \mathrm{H})$, 7.35-7.43 (m, 6H), 7.63-7.67 (m, 4H) ; HRMS calcd for $\mathrm{C}_{22} \mathrm{H}_{25} \mathrm{Br}^{81} \mathrm{BrO}_{2} \mathrm{Si}\left(\mathrm{M}^{+}-t-\mathrm{C}_{4} \mathrm{H}_{9}\right) m / z$ 508.9970, found 508.9974.

\section{(1R,6S,7S,8S)-7-(t-Butyldiphenylsilyloxy)methyl-8-methyl-9-oxabicyclo[4.2.1]nona-2,4-diene}

((+)-24). To a heated $\left(75^{\circ} \mathrm{C}\right)$ stirred solution of $t$-BuOK $(933 \mathrm{mg}, 8.31 \mathrm{mmol})$ in $t$-BuOH $(20 \mathrm{~mL})$ was added a solution of $\mathbf{2 3}(942 \mathrm{mg}, 1.66 \mathrm{mmol})$ in $t$-BuOH $(5 \mathrm{~mL})$. The mixture was stirred at $75{ }^{\circ} \mathrm{C}$ for 30 min, diluted with $\mathrm{H}_{2} \mathrm{O}(200 \mathrm{~mL})$, and extracted with EtOAc $(100 \mathrm{~mL} \square 3)$. The combined extracts were dried and concentrated in vacuo. The residue was purified by column chromatography on silica gel (EtOAc:hexane, 1:80) to provide $356 \mathrm{mg}(53 \%)$ of (+)-24 as a colorless oil: TLC $R_{f} 0.28$ (EtOAc:hexane, 1:20); [ $]_{\mathrm{D}}{ }^{22}+12.3\left(c 0.840, \mathrm{CHCl}_{3}\right)$; IR $2940 \mathrm{~cm}^{-1} ;{ }^{1} \mathrm{H}$ NMR (270 MHz) $\square 0.93(\mathrm{~d}, 3 \mathrm{H}, J=7.0 \mathrm{~Hz}), 1.04$

\footnotetext{
${ }^{5}$ Byproducts derived from catalyst were removed effectively by treatment with DMSO, see: Ahn, Y. M.; Yang, K.; Georg, G. I. Org. Lett. $2001,3,1411-1413$.
} 
(s, 9H), 2.62-2.78 (m, 2H), $3.62(\mathrm{dd}, 1 \mathrm{H}, J=6.6,10.3 \mathrm{~Hz}), 3.69(\mathrm{dd}, 1 \mathrm{H}, J=8.8,10.3 \mathrm{~Hz}), 4.19(\mathrm{dd}, 1 \mathrm{H}, J$ $=1.5,4.8 \mathrm{~Hz}), 4.55(\mathrm{dd}, 1 \mathrm{H}, J=3.9,5.3 \mathrm{~Hz}), 5.81-5.89(\mathrm{~m}, 2 \mathrm{H}), 6.05-6.22(\mathrm{~m}, 2 \mathrm{H}), 7.35-7.43(\mathrm{~m}, 6 \mathrm{H})$, 7.63-7.67 (m, 4H); ${ }^{13} \mathrm{C}$ NMR (68 MHz) $\square 14.0,19.1,26.5 \square 3,50.2,57.0,62.5,80.7,85.9,124.6,124.7$, $127.7 \square 4,129.6 \square 2,133.6 \square 2,135.5 \square 4,137.8,139.0$; HRMS calcd for $\mathrm{C}_{22} \mathrm{H}_{23} \mathrm{O}_{2} \mathrm{Si}\left(\mathrm{M}^{+}-t-\mathrm{C}_{4} \mathrm{H}_{9}\right) \mathrm{m} / \mathrm{z}$ 347.1467 , found 347.1465 .

(1R,6S,7S,8S)-7-Hydroxymethyl-8-methyl-9-oxabicyclo[4.2.1] nona-2,4-diene $((+)-4) . \quad$ To a cooled $\left(0{ }^{\circ} \mathrm{C}\right)$ stirred solution of $(+)-\mathbf{2 4}(356 \mathrm{mg}, 0.880 \mathrm{mmol})$ in THF $(8 \mathrm{~mL})$ was added $n-\mathrm{Bu}_{4} \mathrm{NF}(1.0 \mathrm{M}$ solution in THF, $1.14 \mathrm{~mL}, 1.14 \mathrm{mmol})$. The mixture was stirred for $2 \mathrm{~h}$ and concentrated in vacuo. The residue was purified by column chromatography on silica gel (EtOAc:hexane, 1:4) to provide $146 \mathrm{mg}$

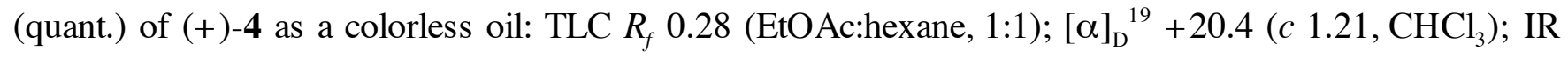
$3400,2940 \mathrm{~cm}^{-1} ;{ }^{1} \mathrm{H}$ NMR $(270 \mathrm{MHz}) \square 1.07(\mathrm{~d}, 3 \mathrm{H}, J=7.0 \mathrm{~Hz}), 1.93(\mathrm{br}, 1 \mathrm{H}, \mathrm{OH}), 2.60-2.79(\mathrm{~m}, 2 \mathrm{H})$, $3.67(\mathrm{dd}, 1 \mathrm{H}, J=7.3,10.3 \mathrm{~Hz}), 3.68(\mathrm{dd}, 1 \mathrm{H}, J=6.2,10.3 \mathrm{~Hz}), 4.23(\mathrm{dd}, 1 \mathrm{H}, J=2.9,5.1 \mathrm{~Hz}), 4.53(\mathrm{dd}$, $1 \mathrm{H}, J=3.5,5.3 \mathrm{~Hz}), 5.82-5.90(\mathrm{~m}, 2 \mathrm{H}), 6.11-6.19(\mathrm{~m}, 2 \mathrm{H}) ;{ }^{13} \mathrm{C} \mathrm{NMR}(68 \mathrm{MHz}) \square 13.8,49.0,57.9,61.7$, 81.3, 85.4, 124.9, 125.1, 137.9, 138.0; HRMS calcd for $\mathrm{C}_{10} \mathrm{H}_{14} \mathrm{O}_{2}\left(\mathrm{M}^{+}\right) \mathrm{m} / z$ 166.0994, found 166.0993.

\section{$(1 S, 2 S, 3 R, 4 R)-2$-(t-Butyldiphenylsilyloxy)methyl-3-(p-toluenesulfonyl)oxymethyl-7-oxa-}

bicyclo[2.2.1] hept-5-ene $((-)-27)$. To a cooled $\left(0^{\circ} \mathrm{C}\right)$ stirred solution of $(-)-7(101 \mathrm{mg}, 0.255 \mathrm{mmol})$ in $\mathrm{CH}_{2} \mathrm{Cl}_{2}(2 \mathrm{~mL})$ were added $\mathrm{Et}_{3} \mathrm{~N}(0.224 \mathrm{~mL}, 2.41 \mathrm{mmol}), \mathrm{TsCl}(72.9 \mathrm{mg}, 0.382 \mathrm{mmol})$, and 4-DMAP (4.4 $\mathrm{mg}, 0.036 \mathrm{mmol})$. The mixture was sitrred for $22 \mathrm{~h}$, diluted with EtOAc $(20 \mathrm{~mL})$, and washed with $\mathrm{H}_{2} \mathrm{O}$ (15 mL $\quad 3)$. The organic layer was dried and concentrated in vacuo. The residue was purified by column chromatography on silica gel (EtOAc:hexane, 1:10) to provide $143 \mathrm{mg}$ (quant.) of (-)-27 as a colorless oil: TLC $R_{f} 0.66$ (EtOAc:hexane, 1:2); [ [0] ${ }_{\mathrm{D}}{ }^{23}-19.1$ (c 1.52, $\mathrm{CHCl}_{3}$ ); IR $2960 \mathrm{~cm}^{-1} ;{ }^{1} \mathrm{H}$ NMR (270 MHz) $\square 1.00(\mathrm{~s}, 9 \mathrm{H}), 1.87-2.05(\mathrm{~m}, 2 \mathrm{H}), 2.40$ (s, 3H), $3.58(\mathrm{~d}, 2 \mathrm{H}, J=7.7 \mathrm{~Hz}), 3.94(\mathrm{t}, 1 \mathrm{H}, J=9.7$ $\mathrm{Hz}), 4.38(\mathrm{dd}, 1 \mathrm{H}, J=4.6,9.7 \mathrm{~Hz}), 4.70(\mathrm{br} \mathrm{s}, 1 \mathrm{H}), 4.78(\mathrm{br} \mathrm{s}, 1 \mathrm{H}), 6.30(\mathrm{dd}, 1 \mathrm{H}, J=1.5,5.8 \mathrm{~Hz}), 6.35$ (dd, $1 \mathrm{H}, J=1.5,5.8 \mathrm{~Hz}), 7.25(\mathrm{~d}, 2 \mathrm{H}, J=8.1 \mathrm{~Hz}), 7.37-7.45(\mathrm{~m}, 6 \mathrm{H}), 7.65-7.69(\mathrm{~m}, 4 \mathrm{H}), 7.71(\mathrm{~d}, 2 \mathrm{H}, J=8.1$ $\mathrm{Hz}$ ); ${ }^{13} \mathrm{C}$ NMR (68 MHz) $\square$ 19.0, 21.6, $26.7 \square 3,39.6,42.7,63.2,70.7,80.2,80.3,127.8 \square 4,129.8 \square 4$, $129.9 \square 2,132.8,133.1 \square 2,135.0,135.4 \square 4,136.0,144.7$; HRMS calcd for $\mathrm{C}_{27} \mathrm{H}_{27} \mathrm{O}_{5} \mathrm{SSi}\left(\mathrm{M}^{+}-t-\mathrm{C}_{4} \mathrm{H}_{9}\right)$ $m / z$ 491.1349, found 491.1340 .

\section{$(1 S, 2 S, 3 S, 4 R)$-2-( $t$-Butyldiphenylsilyloxy)methyl-3-methyl-7-oxabicyclo[2.2.1]hept-5-ene}

((+)-26). To a heated $\left(90^{\circ} \mathrm{C}\right)$ stirred solution of $\mathrm{NaBH}_{4}(98.7 \mathrm{mg}, 2.55 \mathrm{mmol})$ in DMPU $(1 \mathrm{~mL})$ was added a solution of $(-)-27$ obtained above $(143 \mathrm{mg})$ in DMPU $(2 \mathrm{~mL})$. The mixture was sitrred at $90{ }^{\circ} \mathrm{C}$ for $2 \mathrm{~h}$, diluted with EtOAc $(10 \mathrm{~mL})$, and washed with $\mathrm{H}_{2} \mathrm{O}(10 \mathrm{~mL} \mathrm{C})$. The organic layer was dried and concentrated in vacuo. The residue was purified by column chromatography on silica gel (EtOAc:hexane, 1:30) to provide $110 \mathrm{mg}$ (76\% from (-)-7) of (+)-26 as a colorless oil: TLC $R_{f} 0.75$ (EtOAc:hexane, 1:4); $[\square]_{\mathrm{D}}^{22}+1.9\left(c 1.73, \mathrm{CHCl}_{3}\right)$; IR $2940 \mathrm{~cm}^{-1} ;{ }^{1} \mathrm{H} \mathrm{NMR}(270 \mathrm{MHz}) \square 0.95(\mathrm{~d}, 3 \mathrm{H}, J=7.0 \mathrm{~Hz}), 1.06(\mathrm{~s}, 9 \mathrm{H})$, 1.69-1.81 (m, 2H), $3.66(\mathrm{dd}, 1 \mathrm{H}, J=9.7,9.8 \mathrm{~Hz}), 3.77(\mathrm{dd}, 1 \mathrm{H}, J=5.5,9.8 \mathrm{~Hz}), 4.43(\mathrm{~s}, 1 \mathrm{H}), 4.94(\mathrm{~s}, 1 \mathrm{H})$, 6.34 (s, 2H), 7.37-7.45 (m, 6H), 7.65-7.69 (m, 4H); ${ }^{13} \mathrm{C} \mathrm{NMR} \mathrm{(68} \mathrm{MHz)} \square$ 14.4, 19.2, $26.9 \square 3,33.4,42.3$, 63.9, 80.5, 84.9, $127.6 \square 4,129.6 \square 2,133.8,133.9,135.4 \square 2,135.5 \square 4$; HRMS calcd for $\mathrm{C}_{20} \mathrm{H}_{21} \mathrm{O}_{2} \mathrm{Si}$ $\left(\mathrm{M}^{+}-t-\mathrm{C}_{4} \mathrm{H}_{9}\right) \mathrm{m} / z$ 321.1311, found 321.1311 . 
Mixture of $(2 R, 3 S, 4 S, 5 S)-2-[(1 Z)$-Buta-1,3-diene-1-yl]-4-( $t$-butyldiphenylsilyloxy)methyl-3methyl-5-vinyltetrahydrofuran $\quad(25 a), \quad(2 S, 3 S, 4 S, 5 R)-2-[(1 Z)$-Buta-1,3-diene-1-yl]-3-( $t$-butyldiphenylsilyloxy)methyl-4-methyl-5-vinyltetrahydrofuran (25b), and $E$-isomers (25c and 25d) (Entry 1 in Table 1). To a stirred solution of (+)-26 (24.9 mg, $65.8 \square \mathrm{mol})$ in benzene $(6.6 \mathrm{~mL})$ were added 1,3-butadiene (1.0 M solution in benzene, $0.13 \mathrm{~mL}, 0.13 \mathrm{mmol})$ and a solution of $\mathrm{Cl}_{2} \mathrm{Ru}(\mathrm{PCy})_{2}=\mathrm{CHPh}(\mathbf{2 1})(2.7 \mathrm{mg}, 3.3 \square \mathrm{mol})$ in benzene $(0.5 \mathrm{~mL})$. The mixture was stirred for $10 \mathrm{~min}$, diluted with EtOAc $(5 \mathrm{~mL})$, and washed with $\mathrm{H}_{2} \mathrm{O}(3 \mathrm{~mL})$. The organic layer was dried and concentrated in vacuo. The residue was purified by column chromatography on silica gel (EtOAc:hexane, 1:60) to provide $16.8 \mathrm{mg}(60 \%)$ of inseparable mixture of $\mathbf{2 5 a - d}$ as a colorless oil: TLC $R_{f} 0.74$ (EtOAc:hexane, 1:6); IR $2940 \mathrm{~cm}^{-1}$; ${ }^{1} \mathrm{H}$ NMR (270 MHz) $\square 0.96,0.97$ (2d, total 3H, $\left.J=7.0 \mathrm{~Hz}\right), 1.05,1.06,1.07$ (3s, total 9H), 2.03-2.20 (m, 2H), $3.58(\mathrm{~m}, 2 \mathrm{H}), 3.90-4.02,4.35-4.42(2 \mathrm{~m}$, total 1.35H), $4.48(\mathrm{t}, 0.30 \mathrm{H}, J=9.0 \mathrm{~Hz}$, minor $Z$-isomer), 4.87 (ddd, 0.35H, $J=0.9,5.0,8.6 \mathrm{~Hz}$, major $Z$-isomer), 5.02-5.29 (m, 4H), 5.42 (m, $0.30 \mathrm{H}$, minor $Z$-isomer), 5.48 (m, 0.35H, major $Z$-isomer), 5.64-5.93 (m, 1.35H), 5.98-6.38 (m, 1.35H), 6.57-6.73 (m, 0.65H, Z-isomers), 7.35-7.47 (m, 6H), 7.64-7.70 (m, 4H); HRMS calcd for $\mathrm{C}_{28} \mathrm{H}_{36} \mathrm{O}_{2} \mathrm{Si}$ $\left(\mathrm{M}^{+}\right) m / z$ 432.2475, found 432.2485.

Preparation of $(+)-4$ from 25a-d (Entry 2 in Table 2). The following reaction was carried out with bubbling Ar. To a stirred solution of 25a-d $(Z / E=1.8: 1,51.8 \mathrm{mg}, 0.120 \mathrm{mmol})$ in benzene $(40 \mathrm{~mL})$ was added a solution of 2nd generation Grubbs catalyst $22(5.5 \mathrm{mg}, 6.5 \square \mathrm{mol})$ in benzene $(0.5 \mathrm{~mL})$. The mixture was refluxed while each solution of $22(5.5 \mathrm{mg}, 6.5 \square \mathrm{mol})$ in benzene $(0.5 \mathrm{~mL})$ was added three times over a period of 3 days. Then DMSO (92 $\square \mathrm{L}, 1.3 \mathrm{mmol})$ was added to the mixture. After being refluxed for $17 \mathrm{~h}$, the dark solution was passed through a pad of silica gel (EtOAc:hexane, 1:15). The filtered solution was concentrated in vacuo. The residue was purified by column chromatography on silica gel (EtOAc:hexane, 1:80) to provide inseparable mixture of (+)-24 and byproducts (20.3 $\mathrm{mg}$ ).

To a cooled $\left(0{ }^{\circ} \mathrm{C}\right)$ stirred solution of the mixture obtained above $(20.3 \mathrm{mg})$ in THF $(1 \mathrm{~mL})$ was added $n$ $\mathrm{Bu}_{4} \mathrm{NF}(1.0 \mathrm{M}$ solution in THF, $60 \square \mathrm{L}, 60 \square \mathrm{mol})$. The mixture was stirred for $17 \mathrm{~h}$ and concentrated in vacuo. The residue was purified by column chromatography on silica gel (EtOAc:hexane, 1:3) to provide $5.7 \mathrm{mg}(29 \%$ from $\mathbf{2 5 a - d})$ of $(+)-\mathbf{4}$ as a colorless oil.

One-Pot ROM/CM/RCM Reaction of (+)-26 for Preparation of (+) -4. To a stirred solution of 1,3-butadiene (1.0 M solution in benzene, $2.5 \mathrm{~mL}, 2.5 \mathrm{mmol})$ in benzene $(210 \mathrm{~mL})$ was added a solution of $\mathrm{Cl}_{2} \mathrm{Ru}\left(\mathrm{PCy}_{3}\right)_{2}=\mathrm{CHPh}(\mathbf{2 1})(20.9 \mathrm{mg}, 25.4 \square \mathrm{mol})$ in benzene $(0.5 \mathrm{~mL})$. The mixture was stirred for 10 min and a solution of $(+)-26(480 \mathrm{mg}, 1.27 \mathrm{mmol})$ in benzene $(5 \mathrm{~mL})$ was added. After being stirred for $30 \mathrm{~min}$, the mixture was heated to reflux and bubbled a stream of Ar. The mixture was refluxed while each solution of 2nd generation Grubbs catalyst $22(21.5 \mathrm{mg}, 25.4 \square \mathrm{mol})$ in benzene $(0.5 \mathrm{~mL})$ was added five times over a period of 4 days. Then DMSO $(0.56 \mathrm{~mL}, 7.5 \mathrm{mmol})$ was added to the mixture. After being refluxed for $18 \mathrm{~h}$, the dark solution was passed through a pad of silica gel (EtOAc:hexane, 1:15). The filtered solution was concentrated in vacuo. The residue was purified by column chromatography on silica gel (EtOAc:hexane, 1:80) to provide inseparable mixture of (+)-24 and byproducts (175 $\mathrm{mg})$.

To a cooled $\left(0{ }^{\circ} \mathrm{C}\right)$ stirred solution of the mixture obtained above $(175 \mathrm{mg})$ in THF $(3 \mathrm{~mL})$ was added $n$ - 
$\mathrm{Bu}_{4} \mathrm{NF}(1.0 \mathrm{M}$ solution in THF, $1.3 \mathrm{~mL}, 1.3 \mathrm{mmol})$. The mixture was stirred for $80 \mathrm{~min}$ and concentrated in vacuo. The residue was purified by column chromatography on silica gel (EtOAc:hexane, 1:4) to provide $49.0 \mathrm{mg}(23 \%$ from (+)-26) of (+)-4 as a colorless oil.

$(1 S, 2 S, 3 S, 4 R)$-2-Hydroxymethyl-3-methyl-7-oxabicyclo[2.2.1] hept-5-ene $((+)-28)$. To a cooled $\left(0{ }^{\circ} \mathrm{C}\right)$ stirred solution of $(+)-26(127 \mathrm{mg}, 0.335 \mathrm{mmol})$ in THF $(3 \mathrm{~mL})$ was added $n$-Bu $\mathrm{Bu}_{4} \mathrm{NF}(1.0 \mathrm{M}$ solution in THF, $0.40 \mathrm{~mL}, 0.40 \mathrm{mmol}$ ). The mixture was stirred for $3 \mathrm{~h}$ and concentrated in vacuo. The residue was purified by column chromatography on silica gel (EtOAc:hexane, 1:1) to provide $39.3 \mathrm{mg}$ (84\%) of (+)-28 as a colorless oil: TLC $R_{f} 0.32$ (EtOAc:hexane, 1:1); [ $]_{\mathrm{D}}{ }^{22}+18.2$ (c 1.09, $\mathrm{CHCl}_{3}$ ); IR $3400,2940 \mathrm{~cm}^{-1} ;{ }^{1} \mathrm{H}$ NMR (270 MHz) $\square 1.07$ (d, 3H, J = 7.0 Hz), 1.67-1.86 (m, 2H), 2.10 (br, 1H, OH), $3.61(\mathrm{dd}, 1 \mathrm{H}, J=8.8,10.3 \mathrm{~Hz}), 3.81$ (dd, $1 \mathrm{H}, J=5.0,10.3 \mathrm{~Hz}), 4.48$ (br s, 1H), 4.88 (br s, 1H), 6.33 (dd, $1 \mathrm{H}, J=1.5,5.9 \mathrm{~Hz}), 6.37(\mathrm{dd}, 1 \mathrm{H}, J=1.5,5.9 \mathrm{~Hz}) ;{ }^{13} \mathrm{C} \mathrm{NMR}(68 \mathrm{MHz}) \square 14.5,33.6,41.7,62.5,80.6$, 84.7, 135.0, 135.8; HRMS calcd for $\mathrm{C}_{8} \mathrm{H}_{13} \mathrm{O}_{2}\left(\mathrm{M}^{+}+\mathrm{H}\right) \mathrm{m} / \mathrm{z}$ 141.0916, found 141.0914.

One-Pot ROM/CM/RCM Reaction of (+) -28 for Preparation of (+) -4. To a stirred solution of (+)-28 (14.4 mg, $0.103 \mathrm{mmol})$ in benzene $(34 \mathrm{~mL})$ were added 1,3-butadiene (1.0 $\mathrm{M}$ solution in benzene, $0.21 \mathrm{~mL}, 0.21 \mathrm{mmol})$ and a solution of $\mathrm{Cl}_{2} \mathrm{Ru}(\mathrm{PCy})_{2}=\mathrm{CHPh}(\mathbf{2 1})(8.4 \mathrm{mg}, 10 \square \mathrm{mol})$ in benzene $(0.5 \mathrm{~mL})$. After being stirred for $45 \mathrm{~min}$, the mixture was heated to reflux and bubbled a stream of Ar. The mixture was refluxed while each solution of 2nd generation Grubbs catalyst 22 (4.4 mg, $5.1 \square \mathrm{mol})$ in benzene (0.5 $\mathrm{mL}$ ) was added four times over a period of 4 days. Then DMSO (92 $\square \mathrm{L}, 1.3 \mathrm{mmol}$ ) was added to the mixture. After being refluxed for $18 \mathrm{~h}$, the dark solution was passed through a pad of silica gel (EtOAc:hexane, 1:1). The filtered solution was concentrated in vacuo. The residue was purified by column chromatography on silica gel (EtOAc:hexane, 1:4) to provide $3.1 \mathrm{mg}(18 \%)$ of (+) -4 as a colorless oil.

One-Pot ROM/CM/RCM Reaction of (-)-13 for Preparation of $(1 R, 6 S, 7 S, 8 R)-7-(t-B u t y l-$ diphenylsilyloxy)methyl-8-hydroxymethyl-9-oxabicyclo[4.2.1] nona-2,4-diene ((+)-30). To a stirred solution of 1,3-butadiene (1.0 M solution in benzene, $2.8 \mathrm{~mL}, 2.8 \mathrm{mmol})$ in benzene $(70 \mathrm{~mL})$ was added a solution of $\mathrm{Cl}_{2} \mathrm{Ru}\left(\mathrm{PCy}_{3}\right)_{2}=\mathrm{CHPh}(\mathbf{2 1})(17.2 \mathrm{mg}, 20.9 \square \mathrm{mol})$ in benzene $(0.5 \mathrm{~mL})$. The mixture was stirred for $15 \mathrm{~min}$ and a solution of (-)-13 (898 mg, $1.93 \mathrm{mmol})$ in benzene $(5 \mathrm{~mL})$ was added. After being stirred for $30 \mathrm{~min}$, the mixture was heated to reflux and bubbled a stream of Ar. The mixture was refluxed while each solution of 2nd generation Grubbs catalyst 22 (17.8 mg, $20.9 \square \mathrm{mol})$ in benzene (0.5 $\mathrm{mL})$ was added four times over a period of 2 days. Then DMSO $(0.31 \mathrm{~mL}, 4.2 \mathrm{mmol})$ was added to the mixture. After being refluxed for $17 \mathrm{~h}$, the dark solution was passed through a pad of silica gel (EtOAc:hexane, 1:10). The filtered solution was concentrated in vacuo to provide a mixture of crude 29 (d.r. $=c a .1: 1)$ and byproducts $\left(257 \mathrm{mg}\right.$ ), which was used directly in the next step: TLC $R_{f} 0.53$ (EtOAc:hexane, 1:4); ${ }^{1} \mathrm{H}$ NMR (270 MHz) for compound $29 \square 1.05(\mathrm{~s}, 9 \mathrm{H}), 1.11(\mathrm{t}, 3 \mathrm{H} \square 1 / 2, J=7.3 \mathrm{~Hz}$ ), $1.14(\mathrm{t}, 3 \mathrm{H} \square 1 / 2, J=7.0 \mathrm{~Hz}), 1.19(\mathrm{~d}, 3 \mathrm{H} \square 1 / 2, J=5.1 \mathrm{~Hz}), 1.20(\mathrm{~d}, 3 \mathrm{H} \square 1 / 2, J=5.5 \mathrm{~Hz}), 2.74-2.81(\mathrm{~m}$, 2H), 3.29-3.67 (m, 6H), $4.49(\mathrm{~m}, 1 \mathrm{H}), 4.56(\mathrm{q}, 1 \mathrm{H} \square 1 / 2, J=5.5 \mathrm{~Hz}), 4.59$ (q, $1 \mathrm{H} \square 1 / 2, J=5.1 \mathrm{~Hz}), 4.63$ (m, 1H), 5.82-5.89 (m, 2H), 6.08-6.17 (m, 2H), 7.38-7.43 (m, 6H), 7.63-7.66 (m, 4H).

To a stirred solution of the mixture obtained above $(257 \mathrm{mg})$ in $\mathrm{MeOH}(5 \mathrm{~mL})$ was added PPTS (66.8 
mg, $0.266 \mathrm{mmol})$. The mixture was stirred for $4 \mathrm{~h}$, diluted with $\mathrm{H}_{2} \mathrm{O}(20 \mathrm{~mL})$, and extracted with $\mathrm{CH}_{2} \mathrm{Cl}_{2}$ $(20 \mathrm{~mL} \square 3)$. The combined extracts were dried and concentrated in vacuo. The residue was purified by column chromatography on silica gel (EtOAc:hexane, 1:7) to provide $65.5 \mathrm{mg}$ (22\% from (-)-13) of (+)30 as a colorless oil: TLC $R_{f} 0.52$ (EtOAc:hexane, 1:2); [ $]_{\mathrm{D}}{ }^{19}+1.7\left(c 1.45, \mathrm{CHCl}_{3}\right)$; IR 3440, $2930 \mathrm{~cm}^{-1}$; ${ }^{1} \mathrm{H}$ NMR (270 MHz) $\square 1.06$ (s, 9H), 2.63 (br, 1H, OH), 2.82-2.89 (m, 2H), 3.66-3.85 (m, 4H), 4.38 (dd, $1 \mathrm{H}, J=2.6,5.1 \mathrm{~Hz}), 4.47(\mathrm{dd}, 1 \mathrm{H}, J=2.6,5.5 \mathrm{~Hz}), 5.81-5.90(\mathrm{~m}, 2 \mathrm{H}), 6.01-6.15(\mathrm{~m}, 2 \mathrm{H}), 7.36-7.45(\mathrm{~m}$, $6 \mathrm{H}), 7.64-7.68(\mathrm{~m}, 4 \mathrm{H}) ;{ }^{13} \mathrm{C}$ NMR $(68 \mathrm{MHz}) \square 19.1,26.8 \square 3,57.2,57.5,61.3,62.8,81.0,81.1,124.9$, 125.1, $127.8 \square 4,129.9 \square 2,132.9 \square 2,135.5 \square 4,137.7,137.8$; HRMS calcd for $\mathrm{C}_{22} \mathrm{H}_{23} \mathrm{O}_{3} \mathrm{Si}\left(\mathrm{M}^{+}-t-\mathrm{C}_{4} \mathrm{H}_{9}\right)$ $m / z 363.1417$, found 363.1413 .

\section{(1R,6S,7S,8R)-7-(t-Butyldiphenylsilyloxy)methyl-8-( $p$-toluenesulfonyl)oxymethyl-9-oxa-}

bicyclo[4.2.1]nona-2,4-diene ((-)-31). To a cooled $\left(0^{\circ} \mathrm{C}\right)$ stirred solution of (+)-30 (61.6 mg, 0.146 mmol) in $\mathrm{CH}_{2} \mathrm{Cl}_{2}(2 \mathrm{~mL})$ were added $\mathrm{Et}_{3} \mathrm{~N}$ (49 $\left.\square \mathrm{L}, 0.35 \mathrm{mmol}\right)$, TsCl (33.6 mg, $0.176 \mathrm{mmol}$ ), and 4DMAP (8.9 mg, $0.073 \mathrm{mmol})$. The mixture was sitrred for $18 \mathrm{~h}$, diluted with EtOAc $(5 \mathrm{~mL})$, and washed with $1 \mathrm{M}$ aqueous $\mathrm{HCl}(5 \mathrm{~mL})$, saturated aqueous $\mathrm{NaHCO}_{3}(5 \mathrm{~mL})$, and saturated brine $(5 \mathrm{~mL})$, successively. The organic layer was dried and concentrated in vacuo. The residue was purified by column chromatography on silica gel (EtOAc:hexane, 1:15) to provide $85.1 \mathrm{mg}$ (quant.) of (-)-31 as a colorless oil: TLC $R_{f} 0.37$ (EtOAc:hexane, 1:4); [ $]_{\mathrm{D}}{ }^{23}-4.0\left(c 1.66, \mathrm{CHCl}_{3}\right)$; IR $2940 \mathrm{~cm}^{-1} ;{ }^{1} \mathrm{H}$ NMR $(270$ $\mathrm{MHz}) \square 1.00(\mathrm{~s}, 9 \mathrm{H}), 2.41(\mathrm{~s}, 3 \mathrm{H}), 2.76(\mathrm{dq}, 1 \mathrm{H}, J=4.0,7.0 \mathrm{~Hz}), 2.94(\mathrm{~m}, 1 \mathrm{H}), 3.53(\mathrm{dd}, 1 \mathrm{H}, J=7.0,10.0$ $\mathrm{Hz}), 3.60(\mathrm{dd}, 1 \mathrm{H}, J=7.0,10.0 \mathrm{~Hz}), 4.04(\mathrm{t}, 1 \mathrm{H}, J=9.5 \mathrm{~Hz}), 4.09(\mathrm{dd}, 1 \mathrm{H}, J=5.9,9.5 \mathrm{~Hz}), 4.40-4.43(\mathrm{~m}$, 2H), 5.79-5.89 (m, 2H), 5.99-6.06 (m, 2H), $7.25(\mathrm{~d}, 2 \mathrm{H}, J=8.2 \mathrm{~Hz}), 7.36-7.45(\mathrm{~m}, 6 \mathrm{H}), 7.56-7.61(\mathrm{~m}, 4 \mathrm{H})$, $7.70(\mathrm{~d}, 2 \mathrm{H}, J=8.2 \mathrm{~Hz}) ;{ }^{13} \mathrm{C}$ NMR $(68 \mathrm{MHz}) \square 19.1,21.7,26.8 \square 3,54.9,55.5,61.3,68.5,79.6,80.8$, $125.2 \square 2,127.8 \square 4,129.8 \square 2,129.9 \square 4,132.8,132.9,133.0,135.5 \square 4,137.2,137.8,144.7$; HRMS calcd for $\mathrm{C}_{29} \mathrm{H}_{29} \mathrm{O}_{5} \mathrm{SSi}\left(\mathrm{M}^{+}-t-\mathrm{C}_{4} \mathrm{H}_{9}\right) m / z, 517.1505$, found 517.1501 .

Preparation of (+) -24 from (-) -31. To a heated $\left(90{ }^{\circ} \mathrm{C}\right)$ stirred solution of $\mathrm{NaBH}_{4}(78.4 \mathrm{mg}, 2.02$ mmol) in DMPU (1 mL) was added dropwise a solution of (-)-31 (116 mg, $0.202 \mathrm{mmol})$ in DMPU (2 $\mathrm{mL}$ ) over $15 \mathrm{~min}$. The mixture was sitrred at $90{ }^{\circ} \mathrm{C}$ for an additional $10 \mathrm{~min}$, diluted with EtOAc $(5 \mathrm{~mL})$, and washed with $\mathrm{H}_{2} \mathrm{O}(5 \mathrm{~mL} \square 3)$. The organic layer was dried and concentrated in vacuo. The residue was purified by column chromatography on silica gel (EtOAc:hexane, 1:20) to provide $61.9 \mathrm{mg}(76 \%)$ of (+)-24 as a colorless oil.

\section{Mixture of $(1 R, 6 S, 7 S, 8 S)-7-[(1 S)$ - (32) and (1R)-1-(2-Furyl)-1-hydroxymethyl]-8-methyl-9-} oxabicyclo[4.2.1] nona-2,4-diene (33). To a cooled $\left(0{ }^{\circ} \mathrm{C}\right)$ stirred solution of $(+)-4(146 \mathrm{mg}, 0.878$ mmol) in $\mathrm{CH}_{2} \mathrm{Cl}_{2}(3 \mathrm{~mL})$ was added Dess-Martin periodinane $(560 \mathrm{mg}, 1.32 \mathrm{mmol})$. The mixture was stirred for $2 \mathrm{~h}$, diluted with EtOAc $(40 \mathrm{~mL})$, and washed with saturated aqueous $\mathrm{Na}_{2} \mathrm{~S}_{2} \mathrm{O}_{3}(20 \mathrm{~mL} \square 2)$ and saturated aqueous $\mathrm{NaHCO}_{3}(20 \mathrm{~mL})$. The organic layer was dried and concentrated in vacuo to provide crude aldehyde (165 mg), which was used immediately in the next step: TLC $R_{f} 0.57$ (EtOAc:hexane, 1:1); IR 2940, $1720 \mathrm{~cm}^{-1} ;{ }^{1} \mathrm{H}$ NMR $(270 \mathrm{MHz}) \square 1.10$ (d, 3H, $\left.J=6.8 \mathrm{~Hz}\right), 3.15$ (d of quint, $1 \mathrm{H}, J=2.0,6.8 \mathrm{~Hz}$ ), $3.26(\mathrm{ddd}, 1 \mathrm{H}, J=2.4,4.4,6.8 \mathrm{~Hz}), 4.34(\mathrm{dd}, 1 \mathrm{H}, J=2.0,4.9 \mathrm{~Hz}), 5.07$ (dd, $1 \mathrm{H}, J=4.4,5.4 \mathrm{~Hz}), 5.85-$ $5.94(\mathrm{~m}, 2 \mathrm{H}), 6.07-6.19(\mathrm{~m}, 2 \mathrm{H}), 9.69$ (d, 1H, $J=2.4 \mathrm{~Hz})$. 
The following reaction was carried out under Ar. To a cooled $\left(0^{\circ} \mathrm{C}\right)$ stirred solution of crude aldehyde $(165 \mathrm{mg})$ in THF $(6 \mathrm{~mL})$ was added furyllithium $(0.30 \mathrm{M}$ solution in THF, prepared from $n$-BuLi and furan, $3.0 \mathrm{~mL}, 0.90 \mathrm{mmol}$ ). The mixture was stirred at $0{ }^{\circ} \mathrm{C}$ for $40 \mathrm{~min}$, diluted with $5 \%$ aqueous $\mathrm{NaHCO}_{3}$ $(60 \mathrm{~mL})$, and extracted with EtOAc $(30 \mathrm{~mL} \square 3)$. The combined extracts were dried and concentrated in vacuo. The residue was purified by column chromatography on silica gel (EtOAc:hexane, 1:10) to provide $87.9 \mathrm{mg}(43 \%)$ of inseparable mixture of $\mathbf{3 2}$ and $\mathbf{3 3}$ (d.r. $=c a .3: 2$ ) as a pale yellow oil: TLC $R_{f} 0.47$ (EtOAc:hexane, 1:1); IR 3400, $2940 \mathrm{~cm}^{-1}$; ${ }^{1} \mathrm{H}$ NMR (270 MHz) $\square 1.06$ (d, 3H $\square$ 2/5, J = $7.3 \mathrm{~Hz}$ ), 1.21 (d, $3 \mathrm{H} \square 3 / 5, J=7.0 \mathrm{~Hz}$ ), 2.10 (br, 1H, OH), 2.64 (m, $1 \mathrm{H} \square$ 2/5), 2.86-3.06 (m, 2H $\square 3 / 5$ ), 3.01 (m, 1H $\square$ 2/5), $4.14(\mathrm{t}, 1 \mathrm{H} \square 3 / 5, J=5.7 \mathrm{~Hz}), 4.26(\mathrm{dd}, 1 \mathrm{H} \square 2 / 5, J=2.4,4.9 \mathrm{~Hz}), 4.30$ (d, $1 \mathrm{H} \square 3 / 5, J=4.4 \mathrm{~Hz}), 4.66$ (dd, $1 \mathrm{H} \square 3 / 5, J=3.8,10.8 \mathrm{~Hz}), 4.69(\mathrm{~d}, 1 \mathrm{H} \square 2 / 5, J=8.1 \mathrm{~Hz}), 4.79(\mathrm{t}, 1 \mathrm{H} \square 2 / 5, J=4.8 \mathrm{~Hz}), 5.66-5.93(\mathrm{~m}$, $2 \mathrm{H}), 6.08-6.22(\mathrm{~m}, 2 \mathrm{H}), 6.27-6.35(\mathrm{~m}, 2 \mathrm{H}), 7.37$ (m, 1H).

(1R,6S,7R,8S) -7-(Furan-2-carbonyl)-8-methyl-9-oxabicyclo[4.2.1] nona-2,4-diene ((-)-34). To a cooled $\left(0{ }^{\circ} \mathrm{C}\right)$ stirred solution of mixture of 32 and 33 (d.r. $=$ ca. 3:2, $\left.87.9 \mathrm{mg}, 0.383 \mathrm{mmol}\right)$ in $\mathrm{CH}_{2} \mathrm{Cl}_{2}(2$ $\mathrm{mL})$ was added $\mathrm{MnO}_{2}(1.75 \mathrm{~g}, 19.8 \mathrm{mmol})$. The mixture was stirred for $1 \mathrm{~h}$, and insoluble materials were removed by filtration through a Celite-pad and washed well with $\mathrm{CH}_{2} \mathrm{Cl}_{2}$. The combined filtrate and washings were concentrated in vacuo. The residue was purified by column chromatography on silica gel (EtOAc:hexane, 1:8) to provide $63.2 \mathrm{mg}(72 \%)$ of (-)-34 as a colorless oil: TLC $R_{f} 0.53$ (EtOAc:hexane, $1: 1)$; $[\square]_{\mathrm{D}}{ }^{21}-7.0\left(c 0.505, \mathrm{CHCl}_{3}\right)$; IR 2960, $1680 \mathrm{~cm}^{-1} ;{ }^{1} \mathrm{H}$ NMR $(270 \mathrm{MHz}) \square 0.88(\mathrm{~d}, 3 \mathrm{H}, J=7.5 \mathrm{~Hz})$, 3.28 (quint, $1 \mathrm{H}, J=7.5 \mathrm{~Hz}), 4.02(\mathrm{dd}, 1 \mathrm{H}, J=5.5,7.5 \mathrm{~Hz}), 4.35(\mathrm{~d}, 1 \mathrm{H}, J=4.4 \mathrm{~Hz}), 5.32(\mathrm{t}, 1 \mathrm{H}, J=5.5$ Hz), 5.88-5.99 (m, 2H), $6.11(\mathrm{~m}, 1 \mathrm{H}), 6.25(\mathrm{~m}, 1 \mathrm{H}), 6.55(\mathrm{dd}, 1 \mathrm{H}, J=1.5,3.7 \mathrm{~Hz}), 7.24(\mathrm{~d}, 1 \mathrm{H}, J=3.7 \mathrm{~Hz})$, $7.59(\mathrm{~d}, 1 \mathrm{H}, J=1.5 \mathrm{~Hz}) ;{ }^{13} \mathrm{C} \mathrm{NMR}(68 \mathrm{MHz}) \square 15.7,55.9,60.6,76.1,87.1,112.8,117.7,125.3,126.4$, 137.4, 138.7, 146.8, 153.3, 186.1; HRMS calcd for $\mathrm{C}_{14} \mathrm{H}_{14} \mathrm{O}_{3}\left(\mathrm{M}^{+}\right) \mathrm{m} / z$ 230.0943, found 230.0938.

Preparation of (+)-32 from (-) -34. The following reaction was carried out under Ar. To a cooled $\left(-78^{\circ} \mathrm{C}\right)$ stirred solution of $(-)-34(63.2 \mathrm{mg}, 0.207 \mathrm{mmol})$ in THF $(2 \mathrm{~mL})$ was added L-Selectride (1.0 M solution in THF, $1.1 \mathrm{~mL}, 1.1 \mathrm{mmol}$ ). The mixture was stirred at $-78{ }^{\circ} \mathrm{C}$ for $15 \mathrm{~min}$ and $35 \%$ aqueous $\mathrm{H}_{2} \mathrm{O}_{2}(1 \mathrm{~mL})$ was added. After being stirred for $1 \mathrm{~h}$, the mixture was quenched with saturated aqueous $\mathrm{Na}_{2} \mathrm{SO}_{3}(2 \mathrm{~mL})$. The resulting mixture was diluted with $\mathrm{H}_{2} \mathrm{O}(20 \mathrm{~mL})$ and extracted with $\mathrm{CH}_{2} \mathrm{Cl}_{2}(10 \mathrm{~mL}$ $\square$ 5). The combined extracts were dried and concentrated in vacuo. The residue was purified by column chromatography on silica gel (EtOAc:hexane, 1:8) to provide $54.4 \mathrm{mg}(85 \%)$ of (+)-32 as a colorless oil: TLC $R_{f} 0.47$ (EtOAc:hexane, 1:1); [ []$_{\mathrm{D}}{ }^{18}+82.5$ (c 1.85, $\mathrm{CHCl}_{3}$ ); IR 3400, $2940 \mathrm{~cm}^{-1} ;{ }^{1} \mathrm{H}$ NMR $(270$ $\mathrm{MHz}) \square 1.21(\mathrm{~d}, 3 \mathrm{H}, J=7.0 \mathrm{~Hz}), 1.90(\mathrm{~d}, 1 \mathrm{H}, J=3.8 \mathrm{~Hz}, \mathrm{OH}), 2.86-3.06(\mathrm{~m}, 2 \mathrm{H}), 4.14(\mathrm{t}, 1 \mathrm{H}, J=5.7 \mathrm{~Hz})$, $4.30(\mathrm{~d}, 1 \mathrm{H}, J=4.4 \mathrm{~Hz}), 4.66(\mathrm{dd}, 1 \mathrm{H}, J=3.8,10.8 \mathrm{~Hz}), 5.67-5.90(\mathrm{~m}, 2 \mathrm{H}), 6.02-6.08(\mathrm{~m}, 2 \mathrm{H}), 6.28$ (dd, $1 \mathrm{H}, J=0.7,3.3 \mathrm{~Hz}), 6.34(\mathrm{dd}, 1 \mathrm{H}, J=1.8,3.3 \mathrm{~Hz}), 7.38(\mathrm{dd}, 1 \mathrm{H}, J=0.7,1.8 \mathrm{~Hz}) ;{ }^{13} \mathrm{C} \mathrm{NMR}(68 \mathrm{MHz}) \square$ 13.7, 52.1, 56.4, 66.3 , 76.1, 86.4, 106.4, 109.7, 123.9, 124.6, 136.6, 138.2, 141.7, 155.8; HRMS calcd for $\mathrm{C}_{14} \mathrm{H}_{16} \mathrm{O}_{3}\left(\mathrm{M}^{+}\right) \mathrm{m} / z, 232.1099$, found 232.1091.

Preparation of (-) -34 from $(+)-4$ via Weinreb amide 35. To a cooled $\left(0{ }^{\circ} \mathrm{C}\right)$ stirred solution of (+)-4 (21.7 mg, $0.130 \mathrm{mmol})$ in $\mathrm{CH}_{2} \mathrm{Cl}_{2}(1 \mathrm{~mL})$ was added Dess-Martin periodinane (84.5 mg, 0.199 mmol). The mixture was stirred for $1 \mathrm{~h}$, diluted with EtOAc $(2 \mathrm{~mL})$, and washed with saturated aqueous 
$\mathrm{Na}_{2} \mathrm{~S}_{2} \mathrm{O}_{3}(2 \mathrm{~mL} \square 3)$. The organic layer was dried and concentrated in vacuo to provide crude aldehyde (90.2 mg), which was used immediately in the next step.

To a stirred solution of crude aldehyde $(90.2 \mathrm{mg})$ in $t$-BuOH $(2 \mathrm{~mL})$ was added a solution of $\mathrm{NH}_{2} \mathrm{SO}_{3} \mathrm{H}$ (37.8 mg, $0.390 \mathrm{mmol})$ and $\mathrm{Na}_{2} \mathrm{HPO}_{4}(55.4 \mathrm{mg}, 0.390 \mathrm{mmol})$ in $\mathrm{H}_{2} \mathrm{O}(2 \mathrm{~mL})$. Then $\mathrm{NaClO}_{2}(35.3 \mathrm{mg}$, $0.390 \mathrm{mmol}$ ) was added to the mixture. After being stirred for $30 \mathrm{~min}$, the mixture was diluted with $\mathrm{H}_{2} \mathrm{O}$ (4 mL) and extracted with $\mathrm{CH}_{2} \mathrm{Cl}_{2}(2 \mathrm{~mL} \square$ ). The combined extracts were dried and concentrated in vacuo to provide crude acid (102 $\mathrm{mg}$ ), which was used immediately in the next step.

To a stirred solution of crude acid $(102 \mathrm{mg})$ in $\mathrm{CH}_{2} \mathrm{Cl}_{2}(2 \mathrm{~mL})$ were added $\mathrm{Me}(\mathrm{MeO}) \mathrm{NH} \cdot \mathrm{HCl}(50.7 \mathrm{mg}$, $0.520 \mathrm{mmol}$ ), 1-[3-(dimethylamino)propyl]-3-ethylcarbodiimide hydrochloride (49.8 $\mathrm{mg}, 0.260 \mathrm{mmol}), 1$ hydroxybenzotriazole $(35.1 \mathrm{mg}, 0.260 \mathrm{mmol}), 4$-DMAP $(2.0 \mathrm{mg}, 0.016 \mathrm{mmol})$, and $\mathrm{Et}_{3} \mathrm{~N}(0.11 \mathrm{~mL}, 0.78$ mmol). The mixture was stirred for $16 \mathrm{~h}$, diluted with EtOAc $(4 \mathrm{~mL})$, and washed with $\mathrm{H}_{2} \mathrm{O}(4 \mathrm{~mL} \square 3)$. The organic layer was dried and concentrated in vacuo. The residue was purified by column chromatography on silica gel (EtOAc:hexane, 1:5) to provide $49.9 \mathrm{mg}$ of inseparable mixture of $\mathbf{3 5}$ and byproducts: TLC $R_{f} 0.33$ (EtOAc:hexane, 1:1); ${ }^{1} \mathrm{H}$ NMR $(270 \mathrm{MHz}$ ) for compound $35 \square 0.96(\mathrm{~d}, 3 \mathrm{H}, J=$ $7.0 \mathrm{~Hz}), 3.09-3.16(\mathrm{~m}, 2 \mathrm{H}), 3.19(\mathrm{~s}, 3 \mathrm{H}), 3.66(\mathrm{~s}, 3 \mathrm{H}), 4.31(\mathrm{~d}, 1 \mathrm{H}, J=4.8 \mathrm{~Hz}), 5.23(\mathrm{t}, 1 \mathrm{H}, J=5.5 \mathrm{~Hz})$, 5.84-5.92 (m, 2H), $6.05(\mathrm{~m}, 1 \mathrm{H}), 6.24(\mathrm{~m}, 1 \mathrm{H})$.

The following reaction was carried out under Ar. To a cooled $\left(0{ }^{\circ} \mathrm{C}\right)$ stirred solution of the mixture obtained above $(49.9 \mathrm{mg})$ in THF $(2 \mathrm{~mL})$ was added furyllithium $(0.30 \mathrm{M}$ solution in THF, prepared from $n$-BuLi and furan, $1.3 \mathrm{~mL}, 0.39 \mathrm{mmol}$ ). The mixture was stirred at $0{ }^{\circ} \mathrm{C}$ for $40 \mathrm{~min}$, diluted with $5 \%$ aqueous $\mathrm{NaHCO}_{3}(4 \mathrm{~mL})$, and extracted with EtOAc $(2 \mathrm{~mL} \square 3)$. The combined extracts were dried and concentrated in vacuo. The residue was purified by column chromatography on silica gel (EtOAc:hexane, 1:10) to provide $12.8 \mathrm{mg}$ (43\% from (+)-4) of (-)-34 as a colorless oil

\section{$(1 R, 6 S, 7 R, 8 S)-7-[(2 S, 6 R S)-6-H y d r o x y-3-0 x 0-6-h y d r o-2 H$-pyran-2-yl]-8-methyl-9-oxabicyclo-}

[4.2.1] nona-2,4-diene $((+)-36)$. The following reaction was carried out under Ar. To a cooled $\left(0{ }^{\circ} \mathrm{C}\right)$ stirred solution of $(+)-32(23.3 \mathrm{mg}, 0.101 \mathrm{mmol})$ in $\mathrm{CH}_{2} \mathrm{Cl}_{2}(1 \mathrm{~mL})$ were added a solution of $\mathrm{VO}(\mathrm{acac})_{2}$ (4.0 mg, $15 \square \mathrm{mol})$ in $\mathrm{CH}_{2} \mathrm{Cl}_{2}(0.3 \mathrm{~mL})$ and $t$ - $\mathrm{BuOOH}(6.63 \mathrm{M}$ solution in isooctane, $90 \square \mathrm{L}, 0.60 \mathrm{mmol})$. The mixture was stirred for $3 \mathrm{~h}$, quenched with $\mathrm{Me}_{2} \mathrm{~S}(50 \square \mathrm{L})$, and concentrated in vacuo. The residue was purified by column chromatography on silica gel (EtOAc:hexane, 1:3) to provide $22.3 \mathrm{mg}(90 \%)$ of $(+)-36$ (d.r. $=c a$. 1:2) as a colorless oil: TLC $R_{f} 0.24$ (EtOAc:hexane, 1:1); [ $]_{\mathrm{D}}{ }^{19}+80.1\left(c 1.74, \mathrm{CHCl}_{3}\right)$; IR 3350, 2940, $1700 \mathrm{~cm}^{-1}$; ${ }^{1} \mathrm{H}$ NMR $(270 \mathrm{MHz}) \square 1.10$ (d, 3H $\square$ 2/3, J=6.6 Hz), 1.11 (d, 3H $\left.\square 1 / 3, J=7.4 \mathrm{~Hz}\right)$, 2.74-2.91 (m, 2H), 4.06-4.24 (m, 2H), 4.62-4.72 (m, 1H), $5.63(\mathrm{~m}, 1 \mathrm{H})$, 5.80-5.90 (m, 2H), 5.98-6.09 (m, 1H), 6.08 (d, $1 \mathrm{H} \square 2 / 3, J=10.3 \mathrm{~Hz}$ ), 6.13 (dd, $1 \mathrm{H} \square 1 / 3, J=1.5,10.3 \mathrm{~Hz}$ ), 6.37-6.51 (m, 1H), 6.87 (dd, $1 \mathrm{H} \square 2 / 3, J=3.3,10.3 \mathrm{~Hz}), 6.92(\mathrm{dd}, 1 \mathrm{H} \square 1 / 3, J=1.8,10.3 \mathrm{~Hz}) ;{ }^{13} \mathrm{C}$ NMR $(68 \mathrm{MHz})$ signals attributable to the major isomer $\square 14.5,51.5,52.6,73.5,78.1,85.3,87.7,124.3,124.7,127.4,136.5,139.7,144.3$, 196.4, signals attributable to the minor isomer $\square 14.5,52.4,52.9,73.5,77.7,85.4,90.6,124.5,124.7,128.5$, 136.4, 139.5, 147.4, 196.0; HRMS calcd for $\mathrm{C}_{14} \mathrm{H}_{16} \mathrm{O}_{4}\left(\mathrm{M}^{+}\right) \mathrm{m} / z$ 248.1049, found 248.1046.

$(1 R, 6 S, 7 R, 8 S)-7-[(2 S, 6 R)-((+)-37)$ and $(2 S, 6 S)-6-(t$-Butyldimethylsilyloxy)-3-oxo-6-hydro2H-pyran-2-yl]-8-methyl-9-oxabicyclo[4.2.1] nona-2,4-diene $((+)-38)$. To a cooled $\left(0{ }^{\circ} \mathrm{C}\right)$ stirred 
solution of (+)-36 (22.3 mg, $0.091 \mathrm{mmol})$ in $\mathrm{CH}_{2} \mathrm{Cl}_{2}(1 \mathrm{~mL})$ were added $\mathrm{Et}_{3} \mathrm{~N}(0.10 \mathrm{~mL}, 0.72 \mathrm{mmol})$, 4DMAP (6.6 mg, $0.054 \mathrm{mmol}$ ), and TBSCl (54.6 mg, $0.362 \mathrm{mmol})$. The mixture was stirred for $18 \mathrm{~h}$, diluted with saturated brine $(20 \mathrm{~mL})$, and extracted with $\mathrm{CH}_{2} \mathrm{Cl}_{2}(10 \mathrm{~mL} \square 3)$. The combined extracts were dried and concentrated in vacuo. The residue was purified by column chromatography on silica gel (EtOAc:hexane, 1:15) to provide $15.2 \mathrm{mg}(46 \%)$ of $(+)-37$ and $6.1 \mathrm{mg}(19 \%)$ of (+)-38. Compound (+)37 was obtained as a colorless oil: TLC $R_{f} 0.40$ (EtOAc:hexane, 1:3); [ $]_{\mathrm{D}}{ }^{22}+39.4$ (c 1.03, $\mathrm{CHCl}_{3}$ ); IR 2940, $1700 \mathrm{~cm}^{-1}$; ${ }^{1} \mathrm{H}$ NMR (270 MHz) $\square 0.17$ (s, 6H), 0.92 (s, 9H), 1.12 (d, 3H, J=6.6 Hz), 2.76-2.92 (m, $2 \mathrm{H}), 4.07(\mathrm{dd}, 1 \mathrm{H}, J=1.5,10.3 \mathrm{~Hz}), 4.23(\mathrm{~d}, 1 \mathrm{H}, J=4.4 \mathrm{~Hz}), 4.64(\mathrm{t}, 1 \mathrm{H}, J=5.7 \mathrm{~Hz}), 5.62(\mathrm{q}, 1 \mathrm{H}, J=1.5$ $\mathrm{Hz}), 5.78-5.90(\mathrm{~m}, 2 \mathrm{H}), 6.00(\mathrm{~m}, 1 \mathrm{H}), 6.07(\mathrm{dd}, 1 \mathrm{H}, J=1.5,10.3 \mathrm{~Hz}), 6.48(\mathrm{~m}, 1 \mathrm{H}), 6.83(\mathrm{dd}, 1 \mathrm{H}, J=1.5$, $10.3 \mathrm{~Hz}) ;{ }^{13} \mathrm{C}$ NMR (68 MHz) $\square-5.2,-3.9,14.3,17.9,25.6 \square 3,51.9,53.2,77.3,77.9,85.3,91.2,124.1$, 124.7, 127.7, 136.4, 140.2, 149.3, 196.1. Compound (+)-38 was obtained as a colorless oil: TLC $R_{f} 0.50$ (EtOAc:hexane, 1:3); [ $\square]_{\mathrm{D}}{ }^{25}+113\left(c 0.515, \mathrm{CHCl}_{3}\right)$; IR 2940, $1700 \mathrm{~cm}^{-1} ;{ }^{1} \mathrm{H}$ NMR (270 MHz) $\square 0.17$ (s, $6 \mathrm{H}), 0.92(\mathrm{~s}, 9 \mathrm{H}), 1.09(\mathrm{~d}, 3 \mathrm{H}, J=6.6 \mathrm{~Hz}), 2.71-2.87(\mathrm{~m}, 2 \mathrm{H}), 4.22(\mathrm{~d}, 1 \mathrm{H}, J=4.4 \mathrm{~Hz}), 4.61(\mathrm{~d}, 1 \mathrm{H}, J=$ $9.2 \mathrm{~Hz}), 4.72(\mathrm{t}, 1 \mathrm{H}, J=5.9 \mathrm{~Hz}), 5.54(\mathrm{~d}, 1 \mathrm{H}, J=3.3 \mathrm{~Hz}), 5.79-5.90(\mathrm{~m}, 2 \mathrm{H}), 6.00(\mathrm{~m}, 1 \mathrm{H}), 6.01(\mathrm{~d}, 1 \mathrm{H}, J$ $=10.3 \mathrm{~Hz}), 6.47(\mathrm{~m}, 1 \mathrm{H}), 6.78(\mathrm{dd}, 1 \mathrm{H}, J=3.3,10.3 \mathrm{~Hz}) ;{ }^{13} \mathrm{C}$ NMR $(68 \mathrm{MHz}) \square-5.2,-4.6,14.5,17.9$, $25.6 \square 3,51.3,53.2,73.6,77.3,85.4,87.8,124.3,124.6,126.1,136.8,140.1,145.7,196.9$.

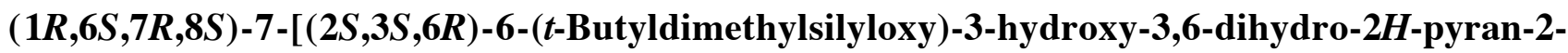
yl]-8-methyl-9-oxabicyclo[4.2.1] nona-2,4-diene $((+)-39)$. To a cooled $\left(0{ }^{\circ} \mathrm{C}\right)$ stirred solution of $(+)$ $37(15.1 \mathrm{mg}, 41.6 \square \mathrm{mol})$ in $\mathrm{MeOH}(0.5 \mathrm{~mL})$ and $\mathrm{CH}_{2} \mathrm{Cl}_{2}(0.5 \mathrm{~mL})$ was added $\mathrm{CeCl}_{3} \cdot 7 \mathrm{H}_{2} \mathrm{O}(94 \mathrm{mg}, 0.25$ mmol). After being stirred at $0{ }^{\circ} \mathrm{C}$ for $10 \mathrm{~min}$, the mixture was cooled to $-78{ }^{\circ} \mathrm{C}$, and then $\mathrm{NaBH}_{4}(9.0 \mathrm{mg}$, $0.25 \mathrm{mmol}$ ) was added. The mixture was stirred at $-78{ }^{\circ} \mathrm{C}$ for $20 \mathrm{~min}$, diluted with saturated brine (20 $\mathrm{mL})$, and extracted with EtOAc (10 mL $\square$ ). The combined extracts were dried and concentrated in vacuo. The residue was purified by column chromatography on silica gel (EtOAc:hexane, 1:10) to provide 13.2

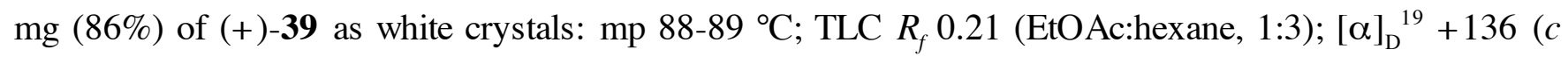
$\left.0.830, \mathrm{CHCl}_{3}\right)$; IR 3380, $2940 \mathrm{~cm}^{-1} ;{ }^{1} \mathrm{H}$ NMR $(270 \mathrm{MHz}) \square 0.14(\mathrm{~s}, 6 \mathrm{H}), 0.91(\mathrm{~s}, 9 \mathrm{H}), 1.09(\mathrm{~d}, 3 \mathrm{H}, J=7.0$ $\mathrm{Hz}), 1.69(\mathrm{~d}, 1 \mathrm{H}, J=10.0 \mathrm{~Hz}, \mathrm{OH}), 2.79-2.95(\mathrm{~m}, 2 \mathrm{H}), 3.53(\mathrm{dd}, 1 \mathrm{H}, J=1.5,10.6 \mathrm{~Hz}), 3.69(\mathrm{~m}, 1 \mathrm{H}), 4.27$ $(\mathrm{d}, 1 \mathrm{H}, J=4.8 \mathrm{~Hz}), 4.35(\mathrm{t}, 1 \mathrm{H}, J=5.8 \mathrm{~Hz}), 5.30(\mathrm{~d}, 1 \mathrm{H}, J=1.1 \mathrm{~Hz}), 5.80(\mathrm{~d}, 1 \mathrm{H}, J=9.9 \mathrm{~Hz}), 5.81-5.93$ $(\mathrm{m}, 2 \mathrm{H}), 6.01-6.14(\mathrm{~m}, 2 \mathrm{H}), 6.30(\mathrm{~m}, 1 \mathrm{H}) ;{ }^{13} \mathrm{C} \mathrm{NMR}(68 \mathrm{MHz}) \square-5.0,-3.9,14.6,18.0,25.7 \square 3,51.6$, 52.7, 63.6, 74.8, 77.5, 86.4, 92.9, 124.6, 125.0, 129.5, 133.4, 136.9, 138.8; HRMS calcd for $\mathrm{C}_{20} \mathrm{H}_{32} \mathrm{O}_{4} \mathrm{Si}$ $\left(\mathrm{M}^{+}\right) m / z$ 364.2070, found 364.2078.

\section{$(1 R, 6 S, 7 R, 8 S)-7-[(2 S, 3 S, 6 R)-3$-Acetoxy-6-(t-butyldimethylsilyloxy)-3,6-dihydro-2H-pyran-2-} yl]-8-methyl-9-oxabicyclo[4.2.1] nona-2,4-diene ((+)-40). To a stirred solution of (+)-39 (22.7 mg, $62.2 \square \mathrm{mol})$ in pyridine $(0.5 \mathrm{~mL})$ and $\mathrm{Ac}_{2} \mathrm{O}(0.5 \mathrm{~mL})$ was added 4-DMAP $(3.4 \mathrm{mg}, 28 \square \mathrm{mol})$. The mixture was stirred for $20 \mathrm{~min}$, diluted with EtOAc $(20 \mathrm{~mL})$, and washed with $1 \mathrm{M}$ aqueous $\mathrm{HCl}(10 \mathrm{~mL})$, saturated aqueous $\mathrm{NaHCO}_{3}(10 \mathrm{~mL})$, and saturated brine $(10 \mathrm{~mL})$, successively. The organic layer was dried and concentrated in vacuo. The residue was purified by column chromatography on silica gel (EtOAc:hexane, 1:15) to provide $22.6 \mathrm{mg}(89 \%)$ of (+)-40 as a colorless oil: TLC $R_{f} 0.39$ (EtOAc:hexane, 1:3); $[\square]_{\mathrm{D}}{ }^{20}+201\left(c 0.835, \mathrm{CHCl}_{3}\right)$; IR 2940, $1730 \mathrm{~cm}^{-1} ;{ }^{1} \mathrm{H}$ NMR $(270 \mathrm{MHz}) \square 0.15(\mathrm{~s}, 6 \mathrm{H}), 0.91(\mathrm{~s}, 9 \mathrm{H})$, 
$1.08(\mathrm{~d}, 3 \mathrm{H}, J=6.6 \mathrm{~Hz}), 2.00(\mathrm{~s}, 3 \mathrm{H}), 2.83-2.99(\mathrm{~m}, 2 \mathrm{H}), 3.66(\mathrm{dd}, 1 \mathrm{H}, J=1.8,10.8 \mathrm{~Hz}), 4.26(\mathrm{t}, 1 \mathrm{H}, J=$ $5.9 \mathrm{~Hz}), 4.27(\mathrm{~d}, 1 \mathrm{H}, J=4.4 \mathrm{~Hz}), 4.87(\mathrm{dt}, 1 \mathrm{H}, J=5.1,1.8 \mathrm{~Hz}), 5.33(\mathrm{~d}, 1 \mathrm{H}, J=1.1 \mathrm{~Hz}), 5.81-5.93(\mathrm{~m}$, 3H), 5.99-6.09 (m, 3H); ${ }^{13} \mathrm{C}$ NMR (68 MHz) $\square-4.9,-3.9,14.2,18.1,21.2,25.8 \square 3,50.6,52.9,65.1,72.9$, 76.4, 86.3, 92.7, 124.4, 124.9, 125.5, 135.3, 137.3, 138.0, 170.8; HRMS calcd for $\mathrm{C}_{18} \mathrm{H}_{25} \mathrm{O}_{5} \mathrm{Si}\left(\mathrm{M}^{+}\right) \mathrm{m} / \mathrm{z}$ 406.2176, found 406.2175.

\section{$(1 R, 6 S, 7 R, 8 S)-7-[(2 S, 3 S, 6 R S)$-3-Acetoxy-6-hydroxy-3,6-dihydro-2H-pyran-2-yl]-8-methyl-9-} oxabicyclo[4.2.1] nona-2,4-diene ((+)-41). A solution of (+)-40 (21.5 mg, 52.9 Ømol) in THF (1 mL) and $2 \mathrm{M}$ aqueous $\mathrm{HCl}(1 \mathrm{~mL})$ was stirred for $1 \mathrm{~h}$. The mixture was diluted with $\mathrm{H}_{2} \mathrm{O}(10 \mathrm{~mL})$ and extracted with $\mathrm{CH}_{2} \mathrm{Cl}_{2}(10 \mathrm{~mL} \square 3)$. The combined extracts were dried and concentrated in vacuo. The residue was purified by column chromatography on silica gel (EtOAc:hexane, 1:2) to provide $15.5 \mathrm{mg}$ (quant.) of (+)-41 (d.r. = ca. 1:8) as white crystals: mp 142-143 ${ }^{\circ} \mathrm{C}$; TLC $R_{f} 0.23$ (EtOAc:hexane, 1:1); $[\square]_{\mathrm{D}}^{21}+304\left(c 0.570, \mathrm{CHCl}_{3}\right)$; IR (KBr) 3420, 2960, $1710 \mathrm{~cm}^{-1} ;{ }^{1} \mathrm{H}$ NMR $(270 \mathrm{MHz})$ for the major isomer $\square 1.14(\mathrm{~d}, 3 \mathrm{H}, J=6.6 \mathrm{~Hz}), 2.01(\mathrm{~s}, 3 \mathrm{H}), 2.79(\mathrm{br}, 1 \mathrm{H}, \mathrm{OH}), 2.80-2.91(\mathrm{~m}, 2 \mathrm{H}), 4.14(\mathrm{dd}, 1 \mathrm{H}, J=2.0$, $10.8 \mathrm{~Hz}), 4.28(\mathrm{t}, 1 \mathrm{H}, J=5.5 \mathrm{~Hz}), 4.29(\mathrm{~d}, 1 \mathrm{H}, J=4.4 \mathrm{~Hz}), 4.84(\mathrm{dd}, 1 \mathrm{H}, J=2.0,5.7 \mathrm{~Hz}), 5.46(\mathrm{br}, 1 \mathrm{H})$, 5.82-5.94 (m, 2H), 6.02-6.09 (m, 2H), $6.05(\mathrm{~d}, 1 \mathrm{H}, J=10.1 \mathrm{~Hz}), 6.20(\mathrm{dd}, 1 \mathrm{H}, J=5.7,10.1 \mathrm{~Hz}) ;{ }^{13} \mathrm{C}$ NMR (68 MHz) for the major isomer $\square 13.8,21.1,50.0,52.7,64.3,67.9,77.5,86.3,88.6,124.4,125.6$, 125.9, 130.7, 137.4, 137.8, 170.6; HRMS calcd for $\mathrm{C}_{16} \mathrm{H}_{20} \mathrm{O}_{5}\left(\mathrm{M}^{+}\right) \mathrm{m} / z$ 292.1311, found 292.1304.

$(1 R, 6 S, 7 R, 8 S)-7-[(2 S, 3 S, 6 S)-((+)-42)$ and $(2 S, 3 R, 6 S)-6-(t$-Butyldimethylsilyloxy)-3-hydroxy3,6-dihydro-2H-pyran-2-yl]-8-methyl-9-oxabicyclo[4.2.1] nona-2,4-diene $((+)-43)$. To a cooled $\left(0{ }^{\circ} \mathrm{C}\right)$ stirred solution of $(+)-38(3.3 \mathrm{mg}, 9.1 \square \mathrm{mol})$ in $\mathrm{MeOH}(1 \mathrm{~mL})$ was added $\mathrm{CeCl}_{3} \cdot 7 \mathrm{H}_{2} \mathrm{O}(20 \mathrm{mg}, 55$ $\square \mathrm{mol})$. The mixture was stirred at $0{ }^{\circ} \mathrm{C}$ for $10 \mathrm{~min}$, and $\mathrm{NaBH}_{4}(1.0 \mathrm{mg}, 27 \square \mathrm{mol})$ was added. After being stirred at $0{ }^{\circ} \mathrm{C}$ for $15 \mathrm{~min}$, the mixture was diluted with saturated brine $(20 \mathrm{~mL})$, and extracted with EtOAc $(10 \mathrm{~mL} \square 5)$. The combined extracts were dried and concentrated in vacuo. The residue was purified by column chromatography on silica gel (EtOAc:hexane, 1:8) to provide $2.1 \mathrm{mg}(63 \%)$ of $(+)-42$ and $1.0 \mathrm{mg}(30 \%)$ of (+)-43. Compound (+)-42 was obtained as white crystals: $\mathrm{mp} 151-152{ }^{\circ} \mathrm{C}$; TLC $R_{f}$

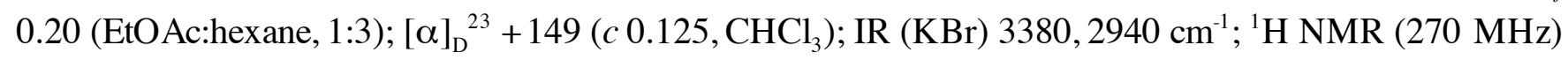
$\square 0.13$ (s, 3H), 0.14 (s, 3H), 0.90 (s, 9H), 1.16 (d, 3H, J = 6.6 Hz), 1.53 (d, 1H, J=10.0 Hz, OH), 2.75$2.88(\mathrm{~m}, 2 \mathrm{H}), 3.65(\mathrm{~m}, 1 \mathrm{H}), 4.01(\mathrm{dd}, 1 \mathrm{H}, J=1.5,10.6 \mathrm{~Hz}), 4.28(\mathrm{~d}, 1 \mathrm{H}, J=4.4 \mathrm{~Hz}), 4.37(\mathrm{t}, 1 \mathrm{H}, J=5.5$ $\mathrm{Hz}), 5.33(\mathrm{~d}, 1 \mathrm{H}, J=2.9 \mathrm{~Hz}), 5.82(\mathrm{dd}, 1 \mathrm{H}, J=2.9,9.9 \mathrm{~Hz}), 5.86-5.95(\mathrm{~m}, 2 \mathrm{H}), 6.06(\mathrm{~m}, 1 \mathrm{H}), 6.14(\mathrm{dd}, 1 \mathrm{H}$, $J=5.9,9.9 \mathrm{~Hz}), 6.31(\mathrm{~m}, 1 \mathrm{H}) ;{ }^{13} \mathrm{C} \mathrm{NMR}(68 \mathrm{MHz}) \square-5.2,-4.5,14.1,18.3,25.7 \square 3,51.2,52.5,62.9$, 69.9, 77.1, 86.3, 89.0, 124.5, 125.4, 128.2, 130.4, 137.3, 138.5; HRMS calcd for $\mathrm{C}_{20} \mathrm{H}_{32} \mathrm{O}_{4} \mathrm{Si}\left(\mathrm{M}^{+}\right) \mathrm{m} / z$ 364.2070, found 364.2071. Compound $(+)-\mathbf{4 3}$ was obtained was a colorless oil: TLC $R_{f} 0.30$

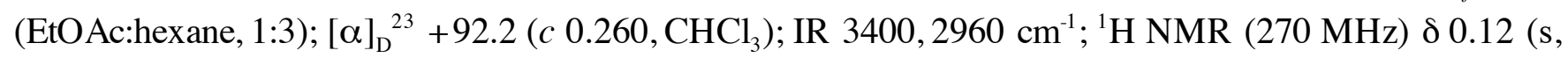
$3 \mathrm{H}), 0.13(\mathrm{~s}, 3 \mathrm{H}), 0.90(\mathrm{~s}, 9 \mathrm{H}), 1.14(\mathrm{~d}, 3 \mathrm{H}, J=7.0 \mathrm{~Hz}), 1.50(\mathrm{~d}, 1 \mathrm{H}, J=10.0 \mathrm{~Hz}, \mathrm{OH}), 2.54(\mathrm{dt}, 1 \mathrm{H}, J=$ 9.5, $6.0 \mathrm{~Hz}), 2.84(\mathrm{~m}, 1 \mathrm{H}), 3.74(\mathrm{t}, 1 \mathrm{H}, J=9.5 \mathrm{~Hz}), 3.94(\mathrm{~m}, 1 \mathrm{H}), 4.21(\mathrm{~d}, 1 \mathrm{H}, J=4.4 \mathrm{~Hz}), 4.82(\mathrm{t}, 1 \mathrm{H}, J=$ $6.0 \mathrm{~Hz}), 5.29(\mathrm{~d}, 1 \mathrm{H}, J=1.1 \mathrm{~Hz}), 5.71(\mathrm{dt}, 1 \mathrm{H}, J=10.3,2.6 \mathrm{~Hz}), 5.82(\mathrm{dd}, 1 \mathrm{H}, J=1.1,10.3 \mathrm{~Hz}), 5.85-5.92$ $(\mathrm{m}, 2 \mathrm{H}), 6.06(\mathrm{~m}, 1 \mathrm{H}), 6.37(\mathrm{~m}, 1 \mathrm{H}) ;{ }^{13} \mathrm{C} \mathrm{NMR}(68 \mathrm{MHz}) \square-5.3,-4.5,13.8,18.3,25.7 \square 3,53.4,55.2$, 68.9, 70.5, 76.8, 85.2, 88.7, 124.8, 125.4, 129.0, 131.5, 137.6, 139.8; HRMS calcd for $\mathrm{C}_{20} \mathrm{H}_{32} \mathrm{O}_{4} \mathrm{Si}\left(\mathrm{M}^{+}\right)$ 
$m / z$ 364.2070, found 364.2067.

Preparation of $(+)-\mathbf{4 1}$ from $(+)-\mathbf{4 2}$. To a stirred solution of $(+)-42(1.9 \mathrm{mg}, 5.2 \square \mathrm{mol})$ in pyridine $(0.5 \mathrm{~mL})$ and $\mathrm{Ac}_{2} \mathrm{O}(0.5 \mathrm{~mL})$ was added 4-DMAP $(2.8 \mathrm{mg}, 23 \square \mathrm{mol})$. The mixture was stirred for $15 \mathrm{~min}$, diluted with EtOAc $(20 \mathrm{~mL})$, and washed with $1 \mathrm{M}$ aqueous $\mathrm{HCl}(10 \mathrm{~mL})$, saturated aqueous $\mathrm{NaHCO}_{3}(10$ $\mathrm{mL})$, and saturated brine $(10 \mathrm{~mL})$, successively. The organic layer was dried and concentrated in vacuo to provide crude acetate $(2.3 \mathrm{mg})$, which was used directly in the next step. The crude acetate was obtained as a colorless oil: TLC $R_{f} 0.52$ (EtOAc:hexane, 1:3); IR 2960, $1730 \mathrm{~cm}^{-1} ;{ }^{1} \mathrm{H}$ NMR $(270 \mathrm{MHz}) \square 0.13$ (s, $\left.3 \mathrm{H}\right)$, $0.15(\mathrm{~s}, 3 \mathrm{H}), 0.90(\mathrm{~s}, 9 \mathrm{H}), 1.14(\mathrm{~d}, 3 \mathrm{H}, J=6.6 \mathrm{~Hz}), 2.00(\mathrm{~s}, 3 \mathrm{H}), 2.79-2.92(\mathrm{~m}, 2 \mathrm{H}), 4.11(\mathrm{dd}, 1 \mathrm{H}, J=2.2$, $10.8 \mathrm{~Hz}), 4.26(\mathrm{t}, 1 \mathrm{H}, J=5.5 \mathrm{~Hz}), 4.27(\mathrm{~d}, 1 \mathrm{H}, J=4.4 \mathrm{~Hz}), 4.81(\mathrm{dd}, 1 \mathrm{H}, J=2.2,5.9 \mathrm{~Hz}), 5.39(\mathrm{~d}, 1 \mathrm{H}, J=$ $2.9 \mathrm{~Hz}), 5.76-5.95(\mathrm{~m}, 3 \mathrm{H}), 6.03-6.14(\mathrm{~m}, 3 \mathrm{H}) ;{ }^{13} \mathrm{C}$ NMR $(68 \mathrm{MHz}) \square-5.3,-4.5,13.8,18.3,21.1,25.7 \square$ 3, 50.2, 52.7, 64.6, 68.1, 77.5, 86.3, 88.8, 124.1, 124.3, 125.6, 132.4, 137.4, 138.0, 170.6.

As described for the preparation of (+)-41 from (+)-40, crude acetate obtained above $(2.3 \mathrm{mg})$ was treated with $2 \mathrm{M}$ aqueous $\mathrm{HCl}(0.5 \mathrm{~mL})$ in THF $(0.5 \mathrm{~mL})$ to provide $1.3 \mathrm{mg}(80 \%$ from $(+)-42)$ of $(+)-\mathbf{4 1}$.

(+)-Mycoepoxydiene (1). To a cooled $\left(0^{\circ} \mathrm{C}\right)$ stirred solution of $(+)-41(14.0 \mathrm{mg}, 47.9 \square \mathrm{mol})$ in $\mathrm{CH}_{2} \mathrm{Cl}_{2}(2 \mathrm{~mL})$ was added $\mathrm{MnO}_{2}(280 \mathrm{mg}, 3.22 \mathrm{mmol})$. The mixture was stirred for $20 \mathrm{~min}$, and insoluble materials were removed by filtration through a Celite-pad and washed well with $\mathrm{CH}_{2} \mathrm{Cl}_{2}$. The combined filtrate and washings were concentrated in vacuo. The residue was purified by column chromatography on silica gel (EtOAc:hexane, 1:3) to provide $10.6 \mathrm{mg}(76 \%)$ of (+)-1 as white crystals: $\mathrm{mp}$ 144-145 ${ }^{\circ} \mathrm{C}$; TLC $R_{f} 0.29$ (EtOAc:hexane, 1:1); [ $]_{\mathrm{D}}{ }^{20}+227$ (c 0.072, MeOH); IR (KBr) 2940, 1740, 1720, $1630 \mathrm{~cm}^{-1}$; ${ }^{1} \mathrm{H}$ NMR $(270 \mathrm{MHz}) \square 1.14(\mathrm{~d}, 3 \mathrm{H}, J=7.0 \mathrm{~Hz}), 2.03(\mathrm{~s}, 3 \mathrm{H}), 2.99-3.10$ (m, 2H), $4.28(\mathrm{t}, 1 \mathrm{H}, J$ $=5.9 \mathrm{~Hz}), 4.32(\mathrm{~d}, 1 \mathrm{H}, J=4.4 \mathrm{~Hz}), 4.49(\mathrm{dd}, 1 \mathrm{H}, J=2.4,11.2 \mathrm{~Hz}), 5.08(\mathrm{dd}, 1 \mathrm{H}, J=2.4,6.0 \mathrm{~Hz}), 5.87-$ $5.95(\mathrm{~m}, 2 \mathrm{H}), 6.01-6.14(\mathrm{~m}, 2 \mathrm{H}), 6.22(\mathrm{~d}, 1 \mathrm{H}, J=9.9 \mathrm{~Hz}), 7.03(\mathrm{dd}, 1 \mathrm{H}, J=6.0,9.9 \mathrm{~Hz}) ;{ }^{13} \mathrm{C}$ NMR $(68$ $\mathrm{MHz}) \square 14.1,20.7,50.0,52.6,63.1,75.9,77.6,86.4,124.4,125.1,126.3,136.9,137.5,140.2,162.2,170.0$; HRMS calcd for $\mathrm{C}_{16} \mathrm{H}_{18} \mathrm{O}_{5}\left(\mathrm{M}^{+}\right) \mathrm{m} / z$ 290.1154, found 290.1155; HPLC analysis (column, Daicel Chiralcel OD+OD-H, $i$-PrOH:hexane = 1:5, flow rate = 0.5 mL/min); $t_{\mathrm{R}}(\mathrm{min})=51.1$ for $(-)-\mathbf{1}, 59.5$ for $(+)-\mathbf{1}$. Compound (+)-1 was determined to be $94 \%$ ee.

4-epi-Mycoepoxydiene ((-) -44). As described for the preparation of (+)-1 from (+)-39, compound $(+)-43$ (4.8 mg, $13 \square \mathrm{mol})$ was converted to $2.5 \mathrm{mg}$ (65\% from (+)-43) of (-)-44. Compound (-)-44 was obtained as a white solid: TLC $R_{f} 0.39$ (EtOAc:hexane, $\left.1: 1\right)$; [ $\left.\square\right]_{\mathrm{D}}{ }^{20}-54.7\left(c 0.055, \mathrm{CHCl}_{3}\right)$; IR 2920, 1740 , $1730,1635 \mathrm{~cm}^{-1} ;{ }^{1} \mathrm{H}$ NMR $(270 \mathrm{MHz}) \square 1.18(\mathrm{~d}, 3 \mathrm{H}, J=7.0 \mathrm{~Hz}), 2.16(\mathrm{~s}, 3 \mathrm{H}), 2.64(\mathrm{dt}, 1 \mathrm{H}, J=10.9,6.6$ Hz), $2.89(\mathrm{~m}, 1 \mathrm{H}), 4.29(\mathrm{~d}, 1 \mathrm{H}, J=4.4 \mathrm{~Hz}), 4.49$ (t, $1 \mathrm{H}, J=5.9 \mathrm{~Hz}), 4.59$ (ddd, 1H, J = 1.1, 3.4, 10.9 Hz), $5.12(\mathrm{dd}, 1 \mathrm{H}, J=3.4,5.1 \mathrm{~Hz}), 5.85-5.95(\mathrm{~m}, 2 \mathrm{H}), 6.06(\mathrm{~m}, 1 \mathrm{H}), 6.13(\mathrm{~m}, 1 \mathrm{H}), 6.19(\mathrm{~d}, 1 \mathrm{H}, J=9.8 \mathrm{~Hz})$, $6.74(\mathrm{ddd}, 1 \mathrm{H}, J=1.1,5.1,9.8 \mathrm{~Hz}) ;{ }^{13} \mathrm{C} \mathrm{NMR}(68 \mathrm{MHz}) \square 14.1,20.8,53.2,53.3,65.9,76.1,79.2,86.1$, 124.6, 124.8, 127.3, 136.6, 137.8, 139.4, 163.0, 171.3; HRMS calcd for $\mathrm{C}_{16} \mathrm{H}_{18} \mathrm{O}_{5}\left(\mathrm{M}^{+}\right) \mathrm{m} / z$ 290.1154, found 290.1154 .

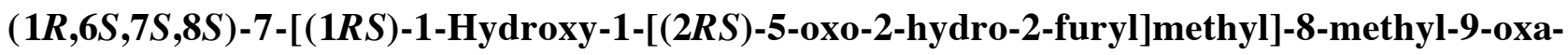
bicyclo[4.2.1]nona-2,4-diene (46). To a cooled $\left(0{ }^{\circ} \mathrm{C}\right)$ stirred solution of (+)-4 (27.5 mg, $\left.0.165 \mathrm{mmol}\right)$ in $\mathrm{CH}_{2} \mathrm{Cl}_{2}(1 \mathrm{~mL})$ was added Dess-Martin periodinane $(210 \mathrm{mg}, 0.496 \mathrm{mmol})$. The mixture was stirred 
for $1 \mathrm{~h}$, diluted with EtOAc ( $5 \mathrm{~mL})$, and washed with saturated aqueous $\mathrm{Na}_{2} \mathrm{~S}_{2} \mathrm{O}_{3}(5 \mathrm{~mL} \square 3)$ and saturated aqueous $\mathrm{NaHCO}_{3}(10 \mathrm{~mL})$. The organic layer was dried and concentrated in vacuo to provide crude aldehyde (29.6 mg), which was used immediately in the next step.

The following reaction was carried out under Ar. To a cooled $\left(-78{ }^{\circ} \mathrm{C}\right)$ stirred solution of crude aldehyde (29.6 mg) in $\mathrm{CH}_{2} \mathrm{Cl}_{2}(1 \mathrm{~mL})$ were added 2-(trimethylsilyloxy)furan (45) (82 $\left.\square \mathrm{L}, 0.50 \mathrm{mmol}\right)$ and TESOTf $(15 \square \mathrm{L}, 0.066 \mathrm{mmol})$. The mixture was stirred at $-78{ }^{\circ} \mathrm{C}$ for $1 \mathrm{~h}$ and TESOTf $(15 \square \mathrm{L}, 0.066$ mmol) was added. The mixture was stirred at $-78^{\circ} \mathrm{C}$ for $40 \mathrm{~min}$ and TESOTf $(7.5 \square \mathrm{L}, 0.033 \mathrm{mmol})$ was added. After being stirred at $-78^{\circ} \mathrm{C}$ for $20 \mathrm{~min}$, the mixture was quenched with $1 \mathrm{M}$ aqueous $\mathrm{HCl}(1 \mathrm{~mL})$, diluted with $\mathrm{H}_{2} \mathrm{O}(5 \mathrm{~mL})$, and extracted with $\mathrm{CH}_{2} \mathrm{Cl}_{2}(5 \mathrm{~mL} 5)$. The combined extracts were dried and concentrated in vacuo. The residue was purified by column chromatography on silica gel (EtOAc:toluene, 1:2) to provide $24.5 \mathrm{mg}(60 \%)$ of $\mathbf{4 6}$ (d.r. $=c a .8: 1)$ as white solids: TLC $R_{f} 0.19$ (EtOAc:toluene, 1:1); IR 3420, 2920, $1750 \mathrm{~cm}^{-1}$; ${ }^{1} \mathrm{H}$ NMR (300 MHz) for major isomer $\square 1.15$ (d, 3H, $J=6.8 \mathrm{~Hz}$ ), 2.39 (br, $1 \mathrm{H}$, $\mathrm{OH}), 2.71-2.83(\mathrm{~m}, 2 \mathrm{H}), 3.92(\mathrm{~m}, 1 \mathrm{H}), 4.26(\mathrm{dd}, 1 \mathrm{H}, J=1.7,4.9 \mathrm{~Hz}), 4.87(\mathrm{t}, 1 \mathrm{H}, J=4.8 \mathrm{~Hz}), 5.02(\mathrm{q}, 1 \mathrm{H}$, $J=1.9 \mathrm{~Hz}), 5.87-5.94(\mathrm{~m}, 2 \mathrm{H}), 6.09-6.20(\mathrm{~m}, 2 \mathrm{H}), 6.22(\mathrm{dd}, 1 \mathrm{H}, J=1.9,5.8 \mathrm{~Hz}), 7.47(\mathrm{dd}, 1 \mathrm{H}, J=1.9,5.8$ $\mathrm{Hz}) ;{ }^{13} \mathrm{C}$ NMR (75 MHz) for major isomer $\square 14.5,50.4,56.3,70.2,79.4,85.0,85.7,122.6,125.3,125.5$, 137.0, 138.1, 154.0, 172.9; HRMS calcd for $\mathrm{C}_{14} \mathrm{H}_{16} \mathrm{O}_{4}\left(\mathrm{M}^{+}\right) \mathrm{m} / z$ 248.1049, found 248.1041.

$(-)-1893 A(2)$ and its $\boldsymbol{E}$-isomer ((-) -47). To a stirred solution of 46 (8.9 $\mathrm{mg}, 0.036 \mathrm{mmol})$ in pyridine $(1 \mathrm{~mL})$ was added $\mathrm{MsCl}(11 \square \mathrm{L}, 0.14 \mathrm{mmol})$. After being stirred for $30 \mathrm{~min}$, the mixture was heated to $80{ }^{\circ} \mathrm{C}$ and stirred for 2 days. The mixture was diluted with EtOAc $(3 \mathrm{~mL})$ and washed with $1 \mathrm{M}$ aqueous $\mathrm{HCl}(3 \mathrm{~mL})$, saturated aqueous $\mathrm{NaHCO}_{3}(3 \mathrm{~mL})$, and saturated brine $(3 \mathrm{~mL})$. The organic layer was dried and concentrated in vacuo. The residue was purified by column chromatography on silica gel (EtOAc:hexane, 1:7) to provide $5.2 \mathrm{mg}(63 \%)$ of (-)-2 and $1.1 \mathrm{mg}(13 \%)$ of (-)-47. Compound (-)-2 was obtained as white crystals: $\mathrm{mp} 91-92{ }^{\circ} \mathrm{C}$; TLC $R_{f} 0.72$ (EtOAc:toluene, $\left.1: 1\right)$; [ []$_{\mathrm{D}}{ }^{22}-293$ ( $c 0.32$, acetone), $[\square]_{\mathrm{D}}{ }^{23}-285$ (c 0.064, acetone); IR (KBr) 2960, 1780, 1750, $1660 \mathrm{~cm}^{-1} ;{ }^{1} \mathrm{H} \mathrm{NMR}\left(300 \mathrm{MHz}, \mathrm{CD}_{3} \mathrm{COCD}_{3}\right)$ $\square 1.01(\mathrm{~d}, 3 \mathrm{H}, J=7.0 \mathrm{~Hz}), 2.85(\mathrm{~m}, 1 \mathrm{H}), 3.71(\mathrm{ddd}, 1 \mathrm{H}, J=3.1,7.5,10.6 \mathrm{~Hz}), 4.26(\mathrm{dd}, 1 \mathrm{H}, J=3.6,5.1$ $\mathrm{Hz}), 4.43(\mathrm{dd}, 1 \mathrm{H}, J=3.1,5.1 \mathrm{~Hz}), 5.56(\mathrm{~d}, 1 \mathrm{H}, J=10.6 \mathrm{~Hz}), 5.85-5.92(\mathrm{~m}, 2 \mathrm{H}), 6.12-6.26(\mathrm{~m}, 2 \mathrm{H}), 6.29$ $(\mathrm{d}, 1 \mathrm{H}, J=5.5 \mathrm{~Hz}), 7.75(\mathrm{~d}, 1 \mathrm{H}, J=5.5 \mathrm{~Hz}) ;{ }^{1} \mathrm{H} \mathrm{NMR}\left(270 \mathrm{MHz}, \mathrm{CDCl}_{3}\right) \square 1.03(\mathrm{~d}, 3 \mathrm{H}, J=7.3 \mathrm{~Hz}), 2.86$ (d of quint., $1 \mathrm{H}, J=3.9,7.3 \mathrm{~Hz}$ ), 3.84 (ddd, 1H, $J=2.4,7.3,10.7 \mathrm{~Hz}), 4.29$ (dd, 1H, $J=3.9,4.9 \mathrm{~Hz}$ ), 4.45 $(\mathrm{dd}, 1 \mathrm{H}, J=2.4,5.1 \mathrm{~Hz}), 5.40(\mathrm{~d}, 1 \mathrm{H}, J=10.7 \mathrm{~Hz}), 5.87-5.96(\mathrm{~m}, 2 \mathrm{H}), 6.08-6.24(\mathrm{~m}, 2 \mathrm{H}), 6.27(\mathrm{~d}, 1 \mathrm{H}, J=$ $5.1 \mathrm{~Hz}), 7.37(\mathrm{~d}, 1 \mathrm{H}, J=5.1 \mathrm{~Hz}) ;{ }^{13} \mathrm{C} \mathrm{NMR}\left(75 \mathrm{MHz}, \mathrm{CD}_{3} \mathrm{COCD}_{3}\right) \square 15.5,52.6,54.8,83.6,85.4,115.4$, 120.1, 125.7, 126.1, 138.1, 138.9, 145.3, 150.9, 170.1; $\left.{ }^{13} \mathrm{C} \mathrm{NMR} \mathrm{(68} \mathrm{MHz,} \mathrm{CDCl}_{3}\right) \square 15.3,50.7,54.8,83.4$, 84.5, 115.3, 119.8, 125.3, 125.6, 136.4, 137.6, 143.5, 149.7, 163.6; HRMS calcd for $\mathrm{C}_{14} \mathrm{H}_{14} \mathrm{O}_{3}\left(\mathrm{M}^{+}\right) \mathrm{m} / \mathrm{z}$ 230.0943, found 230.0942. Compound (-)-47 was obtained as white crystals: mp 112-115 ${ }^{\circ} \mathrm{C}$; TLC $R_{f}$ 0.81 (EtOAc:toluene, 1:1); [] ${ }_{\mathrm{D}}{ }^{23}-148$ (c 0.095, acetone); IR (KBr) 2960, 1750, $1660 \mathrm{~cm}^{-1} ;{ }^{1} \mathrm{H}$ NMR $\left(270 \mathrm{MHz} \mathrm{CDCl}_{3}\right) \square 1.05(\mathrm{~d}, 3 \mathrm{H}, J=7.3 \mathrm{~Hz}), 2.79$ (d of quint., $\left.1 \mathrm{H}, J=3.4,7.3 \mathrm{~Hz}\right), 3.40(\mathrm{ddd}, 1 \mathrm{H}, J=$ $3.4,7.3,11.7 \mathrm{~Hz}), 4.34(\mathrm{dd}, 1 \mathrm{H}, J=3.4,5.1 \mathrm{~Hz}), 4.46(\mathrm{dd}, 1 \mathrm{H}, J=3.4,5.1 \mathrm{~Hz}), 5.86(\mathrm{dd}, 1 \mathrm{H}, J=2.0,11.7$ $\mathrm{Hz}), 5.91-5.98(\mathrm{~m}, 2 \mathrm{H}), 6.11-6.20(\mathrm{~m}, 2 \mathrm{H}), 6.24(\mathrm{dd}, 1 \mathrm{H}, J=2.0,5.4 \mathrm{~Hz}), 7.59(\mathrm{~d}, 1 \mathrm{H}, J=5.4 \mathrm{~Hz}) ;{ }^{13} \mathrm{C}$ NMR (68 MHz, $\left.\mathrm{CDCl}_{3}\right) \square 15.4,52.7,53.5,82.7,85.0,114.5,120.9,125.1,126.0,136.4,137.8,139.1$, 
150.4, 165.9; HRMS calcd for $\mathrm{C}_{14} \mathrm{H}_{14} \mathrm{O}_{3}\left(\mathrm{M}^{+}\right) \mathrm{m} / z$ 230.0943, found 230.0941 .

Preparation of (-) -2 from (-) -47. To stirred solution of (-)-47 (0.8 mg, $3.5 \square \mathrm{mol})$ in pyridine (1 $\mathrm{mL})$ was added $\mathrm{MsCl}(3 \square \mathrm{L}, 35 \square \mathrm{mol})$. The mixture was stirred at $90{ }^{\circ} \mathrm{C}$ for 2 days, diluted with EtOAc $(5 \mathrm{~mL})$, and washed with $\mathrm{H}_{2} \mathrm{O}(3 \mathrm{~mL} \square 2)$ and saturated brine $(3 \mathrm{~mL})$. The organic layer was dried and concentrated in vacuo. The residue was purified by column chromatography on silica gel (EtOAc:hexane, $1: 7)$ to provide $0.5 \mathrm{mg}(63 \%)$ of (-)-2 and $0.2 \mathrm{mg}(25 \%)$ of (-)-47 was recovered.

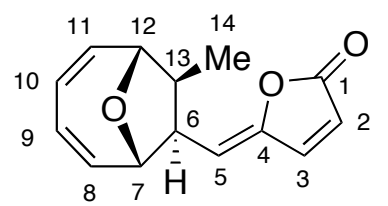

1893A (2)

Table S1. NMR data of natural 1893A (2) and synthetic (-)-2 (in $\mathrm{CD}_{3} \mathrm{COCD}_{3}$ )

\begin{tabular}{|c|c|c|c|c|}
\hline \multirow[b]{2}{*}{ No } & \multicolumn{2}{|c|}{${ }^{13} \mathrm{C} \mathrm{NMR}$} & \multicolumn{2}{|c|}{${ }^{1} \mathrm{H}$ NMR (multiplicity, $J_{\mathrm{H} / \mathrm{H}} \mathrm{Hz}$ ) } \\
\hline & $\begin{array}{c}\text { natural } \\
(125 \mathrm{MHz})^{a}\end{array}$ & $\begin{array}{l}\text { synthetic } \\
(75 \mathrm{MHz})\end{array}$ & natural $(500 \mathrm{MHz})^{a}$ & synthetic $(300 \mathrm{MHz})$ \\
\hline 1 & 170.1 & 170.1 & & \\
\hline 2 & 120.1 & 120.1 & $6.28(\mathrm{~d}, 5.5)$ & $6.29(\mathrm{~d}, 5.5)$ \\
\hline 3 & 145.2 & 145.3 & $7.74(\mathrm{~d}, 5.5)$ & $7.75(\mathrm{~d}, 5.5)$ \\
\hline 4 & 150.9 & 150.9 & & \\
\hline 5 & 115.4 & 115.4 & $5.54(\mathrm{~d}, 11)$ & $5.56(\mathrm{~d}, 10.6)$ \\
\hline 6 & 54.9 & 54.8 & $3.71(\mathrm{ddd}, 3.5,7,11)$ & 3.71 (ddd, 3.1, 7.5, 10.6) \\
\hline 7 & 83.7 & 83.6 & $4.43(\mathrm{dd}, 3.5,5.5)$ & $4.43(\mathrm{dd}, 3.1,5.1)$ \\
\hline 8 & 138.1 & 138.1 & $6.18(\mathrm{dd}, 5.5,8.5)$ & $6.12 \sim 6.26(\mathrm{~m})$ \\
\hline 9 & 126.1 & 126.1 & $5.85(\mathrm{dd}, 7,8.5)$ & $5.85 \sim 5.92(\mathrm{~m})$ \\
\hline 10 & 125.7 & 125.7 & $5.89(\mathrm{dd}, 7,8.5)$ & $5.85 \sim 5.92(\mathrm{~m})$ \\
\hline 11 & 138.9 & 138.9 & $6.23(\mathrm{dd}, 5,8.5)$ & $6.12 \sim 6.26(\mathrm{~m})$ \\
\hline 12 & 85.4 & 85.4 & $4.25(\mathrm{dd}, 3.5,5)$ & $4.26(\mathrm{dd}, 3.6,5.1)$ \\
\hline 13 & 52.5 & 52.6 & $2.83(\mathrm{ddq}, 3.5,7,7)$ & $2.85(\mathrm{~m})$ \\
\hline 14 & 15.5 & 15.5 & $1.02(\mathrm{~d}, 7)$ & $1.01(\mathrm{~d}, 7.0)$ \\
\hline
\end{tabular}

${ }^{a}$ ref) Chen, G.; Lin, Y.; Wen, L.; Vrijmoed, L. L. P.; Jones, E. B. G. Tetrahedron 2003, 59, 4907-4909. 